NBER WORKING PAPER SERIES

\title{
IDENTIFICATION IN ASCENDING AUCTIONS, WITH AN APPLICATION TO DIGITAL RIGHTS MANAGEMENT
}

\author{
Joachim Freyberger \\ Bradley J. Larsen \\ Working Paper 23569 \\ http://www.nber.org/papers/w23569 \\ NATIONAL BUREAU OF ECONOMIC RESEARCH \\ 1050 Massachusetts Avenue \\ Cambridge, MA 02138 \\ July 2017
}

We thank Dominic Coey, Dan Quint, Yoshi Rai, and Caio Waisman for helpful comments. This project was supported by NSF Grant SES-1530632. The views expressed herein are those of the authors and do not necessarily reflect the views of the National Bureau of Economic Research.

NBER working papers are circulated for discussion and comment purposes. They have not been peer-reviewed or been subject to the review by the NBER Board of Directors that accompanies official NBER publications.

(C) 2017 by Joachim Freyberger and Bradley J. Larsen. All rights reserved. Short sections of text, not to exceed two paragraphs, may be quoted without explicit permission provided that full credit, including $\odot$ notice, is given to the source. 
Identification in Ascending Auctions, with an Application to Digital Rights Management Joachim Freyberger and Bradley J. Larsen

NBER Working Paper No. 23569

July 2017

JEL No. C1,C57,D44,L0,L96,O3

\begin{abstract}
$\underline{\text { ABSTRACT }}$
This study provides new identification and estimation results for ascending (traditional English or online) auctions with unobserved auction-level heterogeneity and an unknown number of bidders. When the seller's reserve price and two order statistics of bids are observed, we derive conditions under which the distributions of buyer valuations, unobserved heterogeneity, and number of participants are point identified. We also derive conditions for point identification in cases where reserve prices are binding (in which case bids may be unobserved in some auctions) and present general conditions for partial identification. We propose a nonparametric maximum likelihood approach for estimation and inference. We apply our approach to the online market for used iPhones and analyze the effects of recent regulatory changes banning consumers from circumventing digital rights management technologies used to lock phones to service providers. We find that buyer valuations for unlocked phones dropped after the unlocking ban took effect.
\end{abstract}

\author{
Joachim Freyberger \\ Department of Economics \\ 1180 Observatory Drive \\ Madison, WI 53706 \\ jfreyberger@ssc.wisc.edu \\ Bradley J. Larsen \\ Department of Economics \\ Stanford University \\ 579 Serra Mall \\ Stanford, CA 94305 \\ and NBER \\ bjlarsen@stanford.edu
}

University of Wisconsin-Madison 


\section{INTRODUCTION}

This paper presents an approach to jointly solve two identification challenges to empirical auctions work in ascending auctions: unobserved heterogeneity at the auction level and an unknown number of bidders. Unlike sealed-bid auctions, ascending auctions - both traditional English auctions as well as online auctions - proceed sequentially, and some potential bidders planning to place a bid are not observed doing so. Hence the number of bidders $(N)$ - a key element for identification arguments in empirical auctions methods - is often unobserved to the researcher. Previously proposed solutions to this problem of unknown $N$ rely on the assumption of independent, private values (IPV), and consequently little empirical work exists in English or online auctions with unknown $N$ outside of the IPV framework. The IPV framework does not allow for bidder's values to be correlated through unobserved heterogeneity in the auctioned items, but such unobserved heterogeneity is common in practice. ${ }^{1}$ Previous research has suggested solutions to this challenge of unobserved heterogeneity, but these methods require that the researcher observe $N$ and, furthermore, while useful in first price auctions (where often all bids are observed by the researcher) these methods do not immediately apply to English or online auctions given the incompleteness of bid data in ascending auctions (where the researcher rarely observes all bids - i.e., the thresholds at which each player would drop out of the bidding). In this paper, we provide a unified framework for nonparametric identification and estimation when both problems exist. In particular, we derive conditions for point identification of the distributions of bidder valuations, unobserved heterogeneity, and the number of bidders, as well as partial identification results when these conditions are not met.

We build on the identification arguments of Song (2004), who suggested an approach to handling settings where the number of bidders is unknown and the researcher observes multiple order statistics of bids in English or online auctions. The

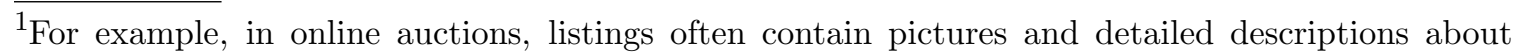
characteristics of the items sold that both the seller and potential buyers can observe, but such information is difficult for the econometrician to quantify. Therefore, items in different auctions can differ dramatically in ways that are observable to the seller and bidders but not to the econometrician.
} 
Song (2004) approach relies on the assumption that bidders have independent private values, in which case the density of a higher order statistic conditional on a lower order statistic will not depend on $N$. We demonstrate that the same argument holds even if bidders valuations are only independent conditional on auction-level heterogeneity and such heterogeneity is unobserved by the econometrician. We also demonstrate that the distribution of the number of bidders is identified.

To nonparametrically identify the distribution of unobserved heterogeneity, we use a similar approach to Li and Vuong (1998), Li, Perrigne, and Vuong (2000), and Krasnokutskaya (2011), relying as they do on the deconvolution result of Kotlarski (1967). These approaches require that the researcher observe two bids that are independent conditional on auction-level unobserved heterogeneity. This approach has been applied to first price auctions in a number of papers (see, for example, Decarolis 2017, Krasnokutskaya and Seim (2011), and others). Various studies (Athey and Haile 2002; Athey, Levin, and Seira 2011; Aradillas-López, Gandhi, and Quint 2013), however, have highlighted that the deconvolution approach to unobserved heterogeneity cannot be applied to English or online auctions using bids alone because bids represent order statistics and not all order statistics are observed, leading to correlation in the observed bids even when individual valuations represent independent draws from the same underlying distribution.

Our approach circumvents this issue of correlated order statistics by relying on an alternative measure of unobserved heterogeneity available to the researcher in many settings. Specifically, we rely on sellers' reserve prices reported to the auction platform. We demonstrate that when reserve prices are secret or non-binding, the distributions of unobserved heterogeneity and buyer valuations are nonparametrically point identified. When reserve prices are public, this can introduce correlation between reserve prices and observed bids, as bids are only recorded if they exceed the public reserve price. We demonstrate how these binding reserve prices affect the likelihood of observed bids and we derive support conditions under which we still obtain point identification. When these conditions are not met, our results yield partial identification. The data requirements for all of our identification arguments, in which the 
researcher is concerned with both unobserved heterogeneity and an unknown number of bidders, are the following: 1) the econometrician observes the seller's reserve price; 2) if reserve prices are secret, the econometrian observes at least two order statistics of bids; and 3) if reserve prices are public, the econometrian observes two order statistics of bids if these exceed the reserve price.

For estimation we propose a nonparametric maximum likelihood approach to jointly estimate the distibutions of unobserved heterogeneity and buyer valuations. Our estimator differs from previous studies relying on the result of Kotlarski (1967), in that these previous studies have generally applied an explicit deconvolution approach, directly estimating a joint characteristic function and then applying inverse Fourier transforms to recover underlying distributions. We take an alternative approach based on nonparametric sieve maximum likelihood estimation (see Gallant and Nychka 1987), which allows us to estimate all parameters in one step. Our framework also nests the possibility of using flexible parametric or semiparametric models for the unknown distributions.

We apply the approach to study the impact of recent legislation regarding consumers circumventing digital rights management. Digital rights management refers to technological locks restricting how consumers use software or hardware. These digital locks are used in computer software, e-books, music, film, cell phones, and in many other products. The US Digital Millenium Copyright Act (DMCA) bans circumvention of these digital locks or production of technologies intended to aid consumers in circumventing digital locks. However, tools and tips for how to cirumvent digital locks are readily available on the Internet, and punishment mechanisms for violators of these laws are not necessarily salient to consumers. Therefore, it is unclear whether the DMCA or related legislation has any effect in practice on market primitives, such as consumers' willingness to purchase — or sellers' willingness to sellpotentially illegally tampered products. Using data from auctions of used iPhones, we analyze the impact of a recent regulatory change banning smartphone unlocking on bidder valuations for unlocked phones. While our application is primarily included as an illustration of our methodology, it provides insights into this previously unstudied 
question. In particular, we find that buyer valuations for unlocked smartphones were lower after the ban was put into place than before. The estimated difference in the means of the distributions of buyer valuations for unlocked smartphones in the pre and post periods corresponds to a decrease in the dollar valuation for the phones of about $27 \%$. This difference suggests that the regulatory change may indeed have had real effects on consumers' willingness to engage in potentially shady behavior.

\section{Related Literature}

This paper contributes to several strands of the empirical auctions literature. First, it extends the approach of Song (2004), which Hortacsu and Nielsen (2010) argued has long been "the standard to beat in the empirical online auctions literature" due to its distinct ability to handle an unknown number of bidders. Other papers building on the Song (2004) approach include Kim and Lee (2014), who developed a test of the independent private values assumption in the Song (2004) environment, and Sailer (2006), who embedded the Song (2004) approach in a repeated auction setting. Other approaches to handling an unknown number of bidders include $\mathrm{An}, \mathrm{Hu}$, and Shum (2010), who considered first price auctions and demonstrated identification when the econometrician observes an instrument for the number of potential bidders (not all of whom necessaily place bids). ${ }^{2}$ Canals-Cerdá and Pearcy (2013), Platt (2015) and Hickman, Hubbard, and Paarsch (2016) provided approaches to estimating the distributions of valuations in online auctions with independent private values, obtaining identification by exploiting the arrival order of bidders. Our nonparametric identification argument is the first of which we are aware for ascending auctions with unobserved heterogeneity and an unknown number of bidders. ${ }^{3}$

\footnotetext{
${ }^{2} \mathrm{Hu}$, McAdams, and Shum (2013) extended the results of An, Hu, and Shum (2010) to apply to settings with non-separable unobserved auction-level heterogeneity (where the number of potential bidders in An, Hu, and Shum (2010) can be considered a form of unobserved heterogeneity in their model) when three bids are observable in first price auctions. Additional work studying unobserved heterogeneity in first price auctions includes Armstrong (2013), and Balat (2015, 2016). As explained above, existing deconvolution approaches (Li and Vuong 1998; Li, Perrigne, and Vuong 2000; Krasnokutskaya 2011) have thus far been applied primarily in first price auctions (with a known number of bidders) where, unlike ascending auctions, independent bids are available.

${ }^{3}$ Canals-Cerdá and Pearcy (2013) provide a parametric identification result that incorporates unobserved heterogeneity.
} 
Second, our paper illustrates the use of variation in potentially binding reserve prices to obtain partial identification. Athey and Haile (2007) discussed several alternative uses of reserve price information. Recent work by Decarolis (2017) applied the Krasnokutskaya (2011) approach using the reserve price and transaction price from first price auctions as two measurements of unobserved heterogeneity, focusing on a sample in which reserve prices were nearly always non-binding in order to avoid the issue of correlation between bids and reserve prices that we address in this paper. Roberts (2013) presented a control function approach to handling unobserved heterogeneity in settings in which the reserve price is monotonic in the unobserved heterogeneity, which does not apply in the settings we study given that reserve prices are chosen by sellers who may have a privately known valuation for the good, and hence reserve prices may not be monotonic in the realization of unobserved auctionlevel heterogeneity.

Third, our approach provides new positive identification results for ascending auctions. As demonstrated in Athey and Haile (2002), even when the number of bidders is known, the joint distribution of bidder valuations is not identified in ascending auctions under arbitrary correlation because the willingness to pay of the highestvaluation bidder is rarely observed (it is never observed in English auctions, but may be observed in some online auction data) and often bids of lower-value bidders are also not observed. Instead of considering arbitrary correlation, we focus on a particular form of correlation among valuations through additively (or multiplicatively) separable auction-level heterogeneity unobserved to the econometrician. Several recent papers have demonstrated that certain objects of interest, such as bounds on optimal reserve prices, or buyer and seller surplus, are identified in ascending auction settings with correlated private values under the assumption that the number of bidders is known (Aradillas-López, Gandhi, and Quint 2013; Coey, Larsen, and Sweeney 2016; Coey, Larsen, Sweeney, and Waisman 2017). Unlike these studies, our approach yields estimates of the underlying valuation distributions, which are useful for studying revenue and welfare under counterfactual auction formats. Quint (2015) provides an alternative argument, related to these papers, that also yields valuation 
identification when the number of bidders is unobserved to the econometrian and when observations are available from auctions under at least two different known probability distributions for the number of bidders.

Our application contributes to a small literature on digital rights management (e.g. Liu, Safavi-Naini, and Sheppard 2003; Von Lohmann 2004; Walker 2003; Stallman 1997) and the literature on piracy and copyright enforcement more broadly (Harbaugh and Khemka 2010). It remains an open question in this literature how effective regulation is at altering consumers' willingness to engage in circumvention. The specific application of cellphone unlocking relates to a variety of previous studies that have examined the role digital locks play in raising switching costs of consumers. This work has focused on non-US markets, including, among others, Tallberg, Hämmäinen, Töyli, Kamppari, and Kivi (2007) (studying Finland), Maicas, Polo, and

Sese (2009) (studying Spain), Nakamura (2010) (studying Japan), and Park and Koo (2016) (studying South Korea). Baker (2007) describes several costs consumers face when unlocking a phone, including time and monetary costs and potential invalidation of the handset's warranty. Farrell and Klemperer (2007) describe general theoretical arguments for how lock-in practices such as handset locking can create inefficiencies and increase firm profits, in particular in settings with network effects such as telecommunications markets. Finally, in focusing our application on smartphones, we contribute to a nacent literature on this industry more broadly. Sinkinson (2014) and Zhu, Liu, and Chintagunta (2015) examine exclusive contracting deals between Apple and AT\&T. Fan and Yang (2016) provides a broad study of the welfare effects of product proliferation and competition in the smartphone industry.

\section{IDENTIFICATION}

2.1. Introduction of Model. We analyze static, single-unit ascending auctions where bidders have symmetric private values. For each bidder $i$, we specify the value of bidder $i$ to take the following form:

$$
V_{i}=X+U_{i}
$$


In many settings, as in our empirical application, the researcher may prefer to model valuations in a multiplicative form, $V_{i}=e^{X} e^{U_{i}}$ and work with logs; all our results hold under multiplicative separability as well, but we state the additively separable version here for ease of exposition. The random variable $X$ is independent of $U_{i}$ for all $i$ and represents a common component through which bidders' valuations (and the seller's reserve price, described below) are correlated. This $X$ is observed by bidders and the seller but is unobserved to the econometrician. Let $U_{i} \sim F_{U}$ with density $f_{U}$, $X \sim F_{X}$ with density $f_{X}$, and $V_{i} \sim F_{V}$ with density $f_{V}$. We assume bidders follow the weakly dominant strategy of bidding their valuations, and hence we will refer to bids and valuations interchangeably. ${ }^{4}$ Let $B_{i}$ be the bid of bidder $i$.

Let $N$ be a random variable with realizations $n$ representing the number of bidders in an auction. We assume $N$ is independent of $X$ and $U$. Let $U^{j}$ refer to the $j^{\text {th }}$ highest $U$ in an auction with at least $j$ bidders but unconditional on the actual realization of the number of bidders $N$. We refer to $U^{j}$ as the $j^{\text {th }}$ unconditional order statistic, to distinguish it from the traditional use of the term order statistic, which refers to an order statistic conditional on a realization or $N=n .^{5}$ Thus, $U^{1}$ is the maximum unconditional order statistic, $U^{2}$ is the second unconditional order statistic, etc., and similarly for order statistics of other random variables (e.g. $B$ and $V$ ).

We allow for the seller's reserve price to be either secret or public. To be precise, in this paper a reserve price is termed to be public if the auction is such that only bids exceeding the reserve price are recorded, and a reserve price is termed to be secret if

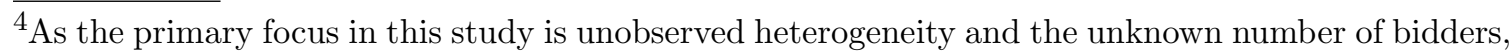
we do not focus on bidders potentially bidding below their values as in Haile and Tamer (2003).

${ }^{5}$ We use this notation and terminology, rather than the traditional notation $U^{n: n}, U^{n-1: n}$, etc., because in our case the order statistics come from samples of varying sizes. That is, $U^{j}$ is the $j^{\text {th }}$ highest $U$ among $N$ bidders, unconditional on the realization of the random variable $N$, and is thus a draw from the distribution

$$
F_{U^{j}}(u) \equiv \sum_{n} \operatorname{Pr}(N=n \mid N \geq j) F_{U^{n-j+1: n}}(u)
$$
}

where $F_{U^{n-j+1: n}}$ is the distribution of the $j^{\text {th }}$ highest bid conditional on $N=n$, which, given that draws of $U$ are i.i.d., is given by the following (see David and Nagaraja 2003):

$$
F_{U^{n-j+1: n}}(u) \equiv\left[\sum_{k=n-j+1}^{n}\left(\begin{array}{l}
n \\
k
\end{array}\right) F_{U}(u)^{k}\left(1-F_{U}(u)\right)^{n-k}\right]
$$


the auction is such that bids need not exceed the reserve price in order to be recorded. We specify the seller's reserve price as

$$
R=X+W
$$

We assume that $W$ is independent of $(X, U, N)$. Let $W \sim F_{W}$ with density $f_{W}$ and $R \sim F_{R}$ with density $f_{R}$. We do not directly model the seller's valuation or choice of reserve price (nor assume that these reserve prices are optimal), but rather simply assume reserve prices take the above form, as in Decarolis (2017). Roberts (2013) takes a different approach, assuming away the seller-specific term $W$ and assuming instead that $R$ is an unknown monotonic function of $X$. Under standard auction rules, optimality of the reserve price combined with an additively (or multiplicatively) separable valuation for the seller would be sufficient conditions for the reserve price to take the form we assume. ${ }^{6}$

We assume the econometrician observes realizations of $R$. In the secret reserve price case, we assume the econometrician also observes at least two unconditional order statistics of bids, $B^{j}$ and $B^{k}$, with $j<k$. In the public reserve price case, the econometrian observes $B^{j}$ and $B^{k}$ if these exceed $R$. In other words, in the secret reserve price case, we assume that at least $k$ bidders wish to place a bid and indeed do place a bid, whereas, in the public reserve price case, at least $k$ bidders wish to place a bid but may be prevented from doing so if the reserve price exceeds their valuation. The econometrician does not observe realizations of $X, U, W$, or $N$. We demonstrate identification of the distributions of each of these random variables. We

${ }^{6}$ If the auction rules are such that the highest bidder wins the good if and only if $B^{1} \geq R$, paying $R$ when $B^{1} \geq R>B^{2}$ and paying $B^{2}$ otherwise, then the optimal reserve price for a seller of value $X+S$, where $S$ is independent of $X$ and $U_{i}$ for all $i$, would satisfy

$$
\begin{aligned}
R & =X+S+\frac{1-F_{V}(R)}{f_{V}(R)} \\
& =X+S+\frac{1-F_{U}(R-X)}{f_{U}(R-X)}
\end{aligned}
$$

Letting $W$ be the random variable such that $W=S+\frac{1-F_{U}(W)}{f_{U}(W)}$ will yield the form $R=X+W$, as above, with $W$ independent of $X$ and $U$. A common alternative rule for ascending auctions (e.g. Larsen 2014) with secret reserve prices is that the highest bidder wins the good if and only if $B^{2}>R$ and pays $B^{2}$. In this case a seller with value $X+S$ would optimally choose a reserve price of $X+S$, yielding again the form $R=X+W$ above, with $W=S$. 
describe three main identification results: First, we obtain point identification when reserve prices are secret. Second, we obtain point identification when reserve prices are public and a support condition is satisfied. Third, we obtain partial identification when reserve prices are public and the support condition is not satisfied.

Throughout, we will let subscripts $j$ and $k$, with $j<k$, be any fixed, positive integers. We denote the lower bound of the support of any random variable $Y$ by $\underline{Y}$ and the upper bound by $\bar{Y}$. Notice that $\underline{V^{j}}=\underline{V^{k}}=\underline{V}$ and $\overline{V^{j}}=\overline{V^{k}}=\bar{V}$ because $V^{j}$ and $V^{k}$ are unconditional order statistics from the same distribution as $V$, and similarly for the supports of $U, U^{j}$, and $U^{k}$. Let $\phi_{Y}(t)$ denote the characteristic function of a random variable $Y$.

We summarize the key assumptions from our discussion thus far in Assumption 1.

Assumption 1. (i) For an auction with $n$ bidders, $R=X+W$ and $V_{i}=X+U_{i}$ for $i=1, \ldots n$, where $X, W, U_{1}, \ldots, U_{n}$ are mutually independent, (ii) $N$ is independent of $X, W$, and $U_{i}$ for all $i=1, \ldots, N$, (iii) $B^{j}=V^{j}$ and $B^{k}=V^{k}$.

We also assume the following:

Assumption 2. $E\left[\left|B^{j}\right|+\left|B^{k}\right|+|R|\right]<\infty$ and $E[X]=0$.

Assumption 3. $(i) \phi_{W}$ and $\phi_{X}$ have only isolated real zeros. (ii) The real zeros of $\phi_{U^{j}}$ and $\phi_{U^{j}}^{\prime}$ are disjoint.

Notice that the means of $X, W, U$, are not identified without a location normalization. To see why let $\tilde{X}=X-c, \tilde{W}=W+c, \tilde{U}_{i}=U_{i}+c$. Then $V_{i}=\tilde{X}+\tilde{U}_{i}$ and $R=\tilde{X}+\tilde{W}$. To identify the distributions we therefore impose in Assumption 2 that $E[X]=0$, but normalizing the mean of $W$ instead yields analogous results. The other moment condition in Assumption 2 is a mild regularity condition and Assumption 3 imposes technical conditions on characteristic functions, which are satisfied by standard distributions. These type of conditions are common in models with multiple measurements; see, for example, Li and Vuong (1998). 
2.2. Identification with Secret Reserve Prices. In the case of secret reserve prices, when the econometrician observes $B^{j}, B^{k}$, and $R$, we obtain the following result:

Theorem 1. Suppose that Assumptions 1-3 hold. Then $F_{X}, F_{W}$, and $F_{U}$ are identified from the joint distribution of bids $B^{j}$ and $B^{k}$ and secret reserve prices $R$. If the number of points of support of $N$ is finite and $F_{U}$ is continuous, then the distribution of $N$ is identified as well.

The theorem implicitly assumes that we always observe $B^{j}$ and $B^{k}$, which implies that $N \geq k$. We could allow for the possibility that realizations of $N$ may be less than $k$, in which case we have to assume that, whenever $N \geq k$, we always observe $B^{j}$ and $B^{k}$. We then can simply do our analysis conditional on $N \geq k$ and still identify $F_{X}, F_{W}$, and $F_{U}$ because $X, W$, and $U$ are independent of $N$. The distribution of the number of bidders is then identified conditional on $N \geq k$ (that is $P(N=n \mid N \geq k)$ for all $n \geq k$ ) under the assumptions of Theorem 1 .

The formal proof, which is in the appendix, proceeds in three steps. First we use one observed bid and the reserve price to identify the distributions of $X$ and $W$, which follows from an extension of Kotlarski's Lemma; see Kotlarski (1967) and Evdokimov and White (2012). While the formal arguments are more involved, it is easy to see that the first two moments of $X$ and $W$ are identified from $E[X]=0$, $E[W]=E[R], \operatorname{var}(X)=\operatorname{cov}\left(W, U^{j}\right)$, and $\operatorname{var}(W)=\operatorname{var}(R)-\operatorname{var}(X)$. Second, we show that knowledge of the characteristic function of $X$ implies identification of the joint distribution of $U^{j}$ and $U^{k}$. Finally, arguments related to those in Song (2004) then yield identification of the distribution of valuations and the number of bidders. Also notice that since $X, W$, and $U$ are independent, identification of the marginal distributions is equivalent to identification of the joint distribution. The arguments in the proof of the theorem can also be used to demonstrate that it is generally possible to identify a parametric distribution of $N$, even if the support is infinite, for example, if $N$ followed a Poisson distribution. 
2.3. Identification with Public Reserve Prices. In the case of public reserve prices, bids will only be observed if they lie above $R$. Define $D_{1}=\mathbf{1}\left(R>B^{j} \geq B^{k}\right)$, $D_{2}=\mathbf{1}\left(B^{j} \geq R>B^{k}\right)$, and $D_{3}=\mathbf{1}\left(B^{j} \geq B^{k} \geq R\right)$. We assume that the observed data is a random sample from the distribution of $\left(R, D_{1}, D_{2}, D_{3}, B^{j} \cdot\left(D_{2}+D_{3}\right), B^{k} \cdot D_{3}\right)$ with $D_{1}+D_{2}+D_{3}=1$. Notice that we therefore assume that $N \geq k$ and that if $B^{k}$ is not observed, then $B^{k}<R$. Point identification is still achieved in this case as long as the support of $B$ is greater than that of $R$ in the strong set order, as we state in the following result.

Theorem 2. Suppose Assumptions 1, 2, and 3(i) hold and that $\bar{R} \leq \bar{B}<\infty$. Then $F_{X}, F_{W}$, and $P\left(U \leq u_{j} \mid U \geq u_{k}\right)$ for all $u_{j}, u_{k} \geq \underline{W}$ are identified from the joint distribution of $\left(R, D_{1}, D_{2}, D_{3}, B^{j} \cdot\left(D_{2}+D_{3}\right), B^{k} \cdot D_{3}\right)$. Moreover, if in addition $\underline{B} \geq$ $\underline{R}>-\infty$, then (i) $F_{U}$ is identified and (ii) the distribution of $N$ is identified if $N$ has finite support and $F_{U}$ is continuous.

The intuition for the identification result is as follows. By the additivity assumption and independence, conditioning on $B^{j}=\bar{B}$ is equivalent to conditioning on $X=\bar{X}$ and $U^{j}=\bar{U}$. Since $W$ is independent of $X$ and $U$, it follows that

$$
\begin{aligned}
P\left(R \leq r \mid B^{j}=\bar{B}\right) & =P\left(R \leq r \mid X=\bar{X}, U^{j}=\bar{U}\right) \\
& =P(W \leq r-\bar{X}) .
\end{aligned}
$$

Hence, the distribution of $W$ is identified up to a location shift, which is fixed by the assumption that $E[R]=E[W]$. Similarly, the joint distribution of $U^{j}$ and $U^{k}$ is identified by considering $P\left(B^{j} \geq b_{j}, B^{k} \geq b_{k} \mid R=\underline{R}\right)$. Finally, using similar arguments as in the proof of Theorem 1 we can then show identification of the distributions of $X, U$, and $N$. If $\underline{B}<\underline{R}$, however, then only $P\left(U \leq u_{j} \mid U \geq u_{k}\right)$ for all $u_{j}, u_{k} \geq \underline{W}$ is identified, but we cannot point identify $P(U \leq u)$.

\section{Estimation And Inference}

In this section we discuss estimation of the unknown densities $f_{X}, f_{W}, f_{U}$, and $f_{U^{k}}$ in both the secret and the public reserve price cases using a nonparametric or 
semiparametric maximum likelihood approach. Our approach does not require estimating $P(N=n)$. We also describe an inference procedure for certain features of the auctions, which is robust to a lack of point identification. Finally, we describe our specific recommendation of a nonparametric or semiparametric estimator.

3.1. Estimation and Inference in the Secret Reserve Case. In the secret reserve price case, the likelihood of the joint distribution of $B^{j}, B^{k}$ and $R$ can be obtained by first writing

$$
\begin{aligned}
P\left(B^{j} \leq b_{j}, B^{k} \leq\right. & \left.b_{k}, R \leq r\right) \\
& =\int P\left(B^{j} \leq b_{j}, B^{k} \leq b_{k}, R \leq r \mid X=x\right) f_{X}(x) d x \\
& =\int P\left(U^{j} \leq b_{j}-x, U^{k} \leq b_{k}-x, W \leq r-x \mid X=x\right) f_{X}(x) d x \\
& =\int P\left(U^{j} \leq b_{j}-x, U^{k} \leq b_{k}-x\right) P(W \leq r-x) f_{X}(x) d x .
\end{aligned}
$$

The first step uses the law of iterated expectations and the remaining steps the independence assumptions. It follows that

$$
\begin{aligned}
f_{B^{j}, B^{k}, R}\left(b_{j}, b_{k}, r\right) & =\int f_{U^{j}, U^{k}}\left(b_{j}-x, b_{k}-x\right) f_{W}(r-x) f_{X}(x) d x \\
& =\int f_{U^{j} \mid U^{k}}\left(b_{j}-x \mid b_{k}-x\right) f_{U^{k}}\left(b_{k}-x\right) f_{W}(r-x) f_{X}(x) d x
\end{aligned}
$$

Notice that $f_{U^{j} \mid U^{k}}\left(b_{j}-x \mid b_{k}-x\right)$ is a function of $f_{U}$ only. For example, when $j=2$ and $k=3$,

$$
f_{U^{j} \mid U^{k}}\left(b_{j}-x, \mid b_{k}-x\right)=\frac{2\left(1-F_{U}\left(b_{j}-x\right)\right) f_{U}\left(b_{j}-x\right)}{\left(1-F_{U}\left(b_{k}-x\right)\right)^{2}}
$$

Denote the data by $Z_{t}=\left(B_{t}^{j}, B_{t}^{k}, R_{t}\right)$, where $t=1, \ldots, T$ denotes an auction. Let $\theta_{0}=\left(f_{X}, f_{W}, f_{U}, f_{U^{k}}\right) \in \Theta$, where $\Theta$ denotes the parameter space. Define the contribution of an individual auction $t$ to the log-likelihood as

$$
l_{s}\left(\theta_{0}, Z_{t}\right)=\ln \left(\int f_{U^{j} \mid U^{k}}\left(B_{t}^{j}-x \mid B_{t}^{k}-x\right) f_{U^{k}}\left(B_{t}^{k}-x\right) f_{W}\left(R_{t}-x\right) f_{X}(x) d x\right)
$$


where the $s$ subscript on $l_{s}(\cdot)$ denotes the secret reserve price case. Thus, given a random sample of $T$ auctions $\left\{B_{t}^{j}, B_{t}^{k}, R_{t}\right\}_{t=1}^{T}$, we can estimate $\theta_{0}$ by maximum likelihood. Searching over the entire parameter space of densities satisfying certain regularity conditions would prove infeasible. We instead maximize the likelihood over a finite-dimensional approximation. In particular, let $\Theta_{T}$ be a finite-dimensional sieve space of $\Theta$, which depends on the sample size $T$ and has the property that $\theta_{0}$ can be approximated arbitrary well by some element in $\Theta_{T}$ when $T$ is large enough (see Section 3.3 for a specific recommendation, as well as Chen (2007) for an overview on sieve estimation). We can now estimate $\theta_{0}$ by

$$
\hat{\theta}_{s}=\underset{\theta \in \Theta_{T}}{\arg \max } \frac{1}{T} \sum_{t=1}^{T} l_{s}\left(\theta, Z_{t}\right) .
$$

Consistency of the estimator follows from standard arguments and regularity conditions, such as Theorem 3.1 in Chen (2007). Furthermore, we can conduct inference about certain functionals of the densities by inverting a likelihood ratio test. Let $g\left(\theta_{0}\right): \Theta \rightarrow \mathbb{R}$ be some functional of interest, such as moments of the distributions, the optimal reserve price, or bidders' surplus. Suppose we are interested in testing $H_{0}: g\left(\theta_{0}\right)=m$ for some $m \in \mathbb{R}$. Define $L_{s}(\theta)=\sum_{t=1}^{T} l_{s}\left(\theta, Z_{t}\right)$. The likelihood ratio test is based on

$$
\hat{\mathcal{T}}_{s}=2\left(\sup _{\theta \in \Theta_{T}} L_{s}(\theta)-\sup _{\theta \in \Theta_{T}: g(\theta)=m} L_{s}(\theta)\right) .
$$

Shen and Shi (2005) provide conditions under which $\hat{\mathcal{T}}_{s} \stackrel{d}{\rightarrow} \chi_{1}^{2}$ if $H_{0}$ is true. Hence, we reject $H_{0}: g\left(\theta_{0}\right)=m$ if $\hat{\mathcal{T}}_{s}>c_{1-\alpha}$, where $c_{1-\alpha}$ is the $1-\alpha$ quantile of the $\chi_{1}^{2}$ distribution. Finally, let

$$
C I_{g\left(\theta_{0}\right)}=\left\{m \in \mathbb{R}: \text { do not reject } H_{0}: g\left(\theta_{0}\right)=m \text { at level } \alpha\right\} \text {. }
$$

Then, by construction, $C I_{g\left(\theta_{0}\right)}$ is a $1-\alpha$ confidence set for $g\left(\theta_{0}\right)$.

An alternative is to specify the model semiparametrically, for example, by assuming parametric distributions for $X, W, U$ but allowing for a nonparametric distribution of $U^{k}$, thus retaining the flexibility to accommodate an unknown distribution of the 
number of bidders. In such a model, inference about the finite-dimensional parameters can be carried out by using the outer product form of the estimated covariance matrix (see Ackerberg, Chen, and Hahn 2012).

3.2. Estimation and Inference in the Public Reserve Case. If the reserve price is public we can still derive the log-likelihood function. To do so, define

$$
\begin{gathered}
p_{1}(r)=\frac{\partial}{\partial r} P\left(R \leq r, D_{1}=1\right), \\
p_{2}\left(r, b_{j}\right)=\frac{\partial}{\partial b_{j} \partial r} P\left(B^{j} \leq b_{j}, R \leq r, D_{2}=1\right),
\end{gathered}
$$

and

$$
p_{3}\left(r, b_{j}, b_{k}\right)=\frac{\partial}{\partial b_{j} \partial b_{k} \partial r} P\left(B^{j} \leq b_{j}, B^{k} \leq b_{k}, R \leq r, D_{3}=1\right) .
$$

We show in Appendix B that

$$
\begin{gathered}
p_{1}(r)=\int_{-\infty}^{\infty} \int_{-\infty}^{r-x} F_{U^{j} \mid U^{k}}\left(r-x \mid u_{k}\right) f_{U^{k}}\left(u_{k}\right) d u_{k} f_{W}(r-x) f_{X}(x) d x, \\
p_{2}\left(r, b_{j}\right)=\int_{-\infty}^{\infty} \int_{-\infty}^{r-x} f_{U^{j} \mid U^{k}}\left(b_{j}-x \mid u_{k}\right) f_{U^{k}}\left(u_{k}\right) d u_{k} f_{W}(r-x) f_{X}(x) d x
\end{gathered}
$$

and

$$
p_{3}\left(r, b_{j}, b_{k}\right)=\int_{-\infty}^{\infty} f_{U^{j} \mid U^{k}}\left(b_{j}-x \mid b_{k}-x\right) f_{U^{k}}\left(b_{k}-x\right) f_{W}(r-x) f_{X}(x) d x .
$$

Notice that since $f_{U^{j} \mid U^{k}}$ and $F_{U^{j} \mid U^{k}}$ depend on $f_{U}$ only, all three expressions only depend on the four densities $f_{X}, f_{W}, f_{U}$, and $f_{U^{k}}$. Now define

$$
L_{p}\left(\theta_{0}\right)=\sum_{t: D_{1 t}=1} \ln \left(p_{1}\left(R_{t}\right)\right)+\sum_{t: D_{2 t}=1} \ln \left(p_{2}\left(R_{t}, B_{t}^{j}\right)\right)+\sum_{t: D_{3 t}=1} \ln \left(p_{3}\left(R_{t}, B_{t}^{j}, B_{t}^{k}\right)\right)
$$

where $p$ in $L_{p}(\cdot)$ denotes the public reserve price case. Under the condition of Theorem 2 , where $\theta_{0}$ is point identified, and the regularity conditions of Theorem 3.1 in Chen (2007), we can consistently estimate $\theta_{0}$ by

$$
\hat{\theta}_{p}=\underset{\theta \in \Theta_{T}}{\arg \max } L_{p}(\theta) .
$$


Moreover, we can obtain confidence intervals for certain functionals of $\theta_{0}$ by inverting a likelihood ratio test, analogous to the secret reserve price setting, or do inference in a semiparametric setting.

As shown in Theorem 2 and the related discussion, in general $\theta_{0}$ might not be point identified if the reserve price is public. In this case, $\hat{\theta}_{p}$ will not be a consistent estimator of $\theta_{0}$, but we can still conduct inference using a likelihood ratio test. Similar to before, let $g\left(\theta_{0}\right): \Theta \rightarrow \mathbb{R}$ be some functional and suppose we are interested in testing $H_{0}: g\left(\theta_{0}\right)=m$ for some $m \in \mathbb{R}$. The likelihood ratio test is based on

$$
\hat{\mathcal{T}}_{p}=2\left(\sup _{\theta \in \Theta_{T}} L_{p}(\theta)-\sup _{\theta \in \Theta_{T}: g(\theta)=m} L_{p}(\theta)\right) .
$$

If $\theta_{0}$ is not point identified, $\hat{\mathcal{T}}_{p}$ does not converge to a $\chi_{1}^{2}$ in distribution. Nevertheless, we can obtain the critical values by using a weighted bootstrap as shown by Chen, Tamer, and Torgovitsky (2011). While this procedure is very flexible and allows for partial identification under weak assumptions, a downside is that it is computationally very demanding.

3.3. Computational and Practical Details. In our application in Section 4 below, we choose as our sieve space the space of normalized, orthogonal Hermite polynomials, as in Gallant and Nychka (1987). This allows us to flexibly approximate the density function for any random variable $Y \in\left\{W, U^{k}, U, X\right\}$ by

$$
f_{Y}(y) \approx \frac{1}{\sigma}\left(\sum_{k=0}^{K} \theta_{k}^{Y} H_{k}\left(\frac{y-\mu_{Y}}{\sigma_{Y}}\right)\right)^{2} \frac{1}{\sqrt{2 \pi}} e^{-\frac{1}{2}\left(\frac{y-\mu_{Y}}{\sigma_{Y}}\right)^{2}}
$$

where $K$ is a smoothing parameter and $\theta^{Y}, \mu_{Y}$, and $\sigma_{Y}$ are estimated. $H_{k}$ are Hermite polynomials defined by $H_{1}(x)=1, H_{2}(x)=x$, and, for $k>2, H_{k}(x)=$ $\frac{1}{\sqrt{k}}\left[x H_{k-1}(x)-\sqrt{k-1} H_{k-2}(x)\right]$.

Plugging in these approximating polynomials, we maximize the above likelihood expressions subject to the constraints $\sum_{i=1}^{K}\left(\theta_{i}^{Y}\right)^{2}=1$ for each random variable $Y \in\left\{W, U^{k}, U, X\right\}$, which ensures each approximated function is indeed a density 
function, and also subject to the constraint $E[X]=0$. The location and scale pa-

rameters $\left\{\mu_{Y}, \sigma_{Y}\right\}_{Y \in\left\{W, U^{k}, U, X\right\}}$ are not required for consistent estimation but improve the performance of the estimator. We estimate them in a parametric initial step, maximizing the likelihood while fixing the vectors $\theta^{Y}$ to have the first element $\theta_{0}^{Y}=1$ and the remaining elements $\theta_{k}^{Y}=0$ for all $k>0$. That is, each density $f_{Y}$ is approximated by a $N\left(\mu_{Y}, \sigma_{Y}\right)$. We then plug in the estimated values of $\left\{\hat{\mu}_{Y}, \hat{\sigma}_{Y}\right\}_{Y \in\left\{W, U^{k}, U, X\right\}}$ into the likelihood expression and maximize over $\left\{\theta^{Y}\right\}_{Y \in\left\{W, U^{k}, U, X\right\}}$. We perform the integration in the likelihood by Gauss-Hermite quadrature (see Judd 1998).

In the application below, we also present results from estimating the model semiparametrically. In the semiparametric estimation, we specify the distributions of $X$, $U$, and $W$ to be normally distributed and we approximate the density of $U^{k}$ using Hermite polynomials, as above, retaining flexibility to allow for an unknown distribution for the number of bidders.

\section{Application to Used Smartphone Auctions}

\subsection{Background on Digital Rights Management and Smartphone Unlock-}

ing. Digital rights management (DRM) refers to technological locks placed on software or hardware to restrict its use or modification. The use of these locks has been highly controversial. Proponents of DRM argue that these restrictions are necessary to prevent copyright infringement of digital intellectual property (Liu, Safavi-Naini, and Sheppard 2003). Opponents argue that DRM takes a step beyond traditional copyright law by controlling how consumers access or use goods or digital content they have legally purchased, suggesting that these laws instead serve primarily to restrict competition between producers (Von Lohmann 2004; Walker 2003; Stallman 1997). A number of products are controlled through DRM, including, among many others, computer software, with digital locks enforcing limited installs or requiring activation keys; e-books, music, or film, with limits on sharing or on device compatibility; and cellular handsets, with digital locks between the subscriber identification module (SIM) and the phone's software, restricting the handset to only function on a particular provider's cellular service network. 
In the United States, the key law regarding DRM is the Digital Millennium Copyright Act (DMCA) of 1998. This law was implemented in response to the 1996 copyright treaty of the World Intellectual Property Organization, which required its members (including the United States) to adopt measures to prohibit tampering with digital locks. In the early years of the DMCA, cellular handset unlocking was granted an explicit exemption, and consumers could legally unlock their out-ofcontract phones through a variety of do-it-yourself or third-party services (Van Camp 2013). In late 2012, the copyright office of the Library of Congress failed to renew this exemption, arguing that phone unlocking tampers with copyrighted firmware and hence is arguably in violation of the law (Federal Register 2012; Couts 2012). This change made phone unlocking illegal as of January 26, 2013, imposing a fine of $\$ 500,000$ and a sentence of five years in prison for unauthorized unlocking (Wyatt 2013). Contemporary conversations among consumers online suggest that consumers were nervous as to how this massive fine and prison sentence would be enforced and to whom it would apply (Velazco 2013; Khanna 2013). In response to backlash from consumer advocates (Wyatt 2013), a bill was eventually signed into law in 2014 to re-allow consumer unlocking of phones.

Although laws such as the DMCA have arisen to prohibit the production or distribution of technology intended to circumvent these digital locks, these laws and copyright laws in general are notoriously difficult to enforce (Harbaugh and Khemka 2010) and violations are difficult to police. Given this enforcement challenge, it remains an open question whether these laws are effective in altering individuals' (buyers and sellers) willingness to engage in DRM circumvention. We contribute to this question by examining buyers' willingness to pay and sellers' pricing for DRMtampered goods - unlocked smartphones - before and after the January 2013 ban on unlocking. While the purpose of this exercise is primarily to illustrate our method rather than provide a definitive answer to the effects of this regulatory change, the analysis provides several insights that we discuss below. 
4.2. Data on Used Smartphone Auctions. We use a new dataset of eBay auctions for used iPhones. The sample consists of used iPhone 4 and $4 \mathrm{~S}$ models with 8, 16, or 32 gigabytes (GB) of memory and of black or white color. These models were the most frequently auctioned iPhone models during our sample period, September 22, 2012 to May 21, 2013. We choose this sample period as it begins after the introduction of the iPhone 5 (September 21, 2012) and includes the date of the regulatory change banning phone unlocking, January 26, 2013. The models we study were released between June 24, 2010 and October 14, 2011, prior to the start date of our sample, and thus a large number of used (unlocked and locked) iPhone 4 and $4 \mathrm{~S}$ handsets had accumulated and were being sold in our sample period. ${ }^{7}$

As with many real-world ascending-like auctions, the number of bidders is not observed in our setting: potential bidders who arrive at the auction after the standing bid has passed their values will not be observed placing a bid. These auctions also exemplify a setting in which it is important to account for unobserved heterogeneity, as used smartphones can differ dramatically in ways that are observable to the seller and bidders but not to the econometrician, such as through a cracked screen, scratched surface, missing USB adapter, or faulty battery, or in positive ways, such as a lack of wear and tear. ${ }^{8}$ Pictures and detailed descriptions posted by the seller contain information about these characteristics that both the seller and potential buyers can observe, but such information is difficult for the econometrician to quantify. ${ }^{9}$

We focus our application on auctions in which the seller used a secret reserve price. For each auction, the data contains the second and third unconditional order statistics of bids, the seller's secret reserve price, the shipping fee, and an indicator for whether

\footnotetext{
${ }^{7}$ The precise release dates were June 24, 2010 for the black iPhone 416 GB and 32 GB; April 28, 2011 for the white iPhone 416 GB and 32 GB; and October 14, 2011 for either color of the iPhone $48 \mathrm{~GB}$ and either color of the iPhone $4 \mathrm{~S} 16$ or $32 \mathrm{~GB}$. The iPhone $4 \mathrm{~S} 8 \mathrm{~GB}$ was not released until after our sample, on September 20, 2013.

${ }^{8}$ Our focus on used phones is also due to the fact that new unlocked phones are more likely to have been unlocked (legally) by the original vendor, and thus the alteration of the DMCA is less likely to have impacted new phones.

${ }^{9}$ When observable to the econometrican, text descriptions could be exploited using natural language parsing algorithms or images could be analyzed with image processing algorithms, and this could aid in accounting for item-level heterogeneity. In such cases our approach would remain useful to account for remaining unobserved heterogeneity.
} 
the phone was locked to a particular carrier (Verizon or AT\&T) or unlocked. We drop all auctions in which the bids, the reserve price, or the shipping fee lies outside of their respective 0.01 and 0.99 quantiles. We also drop auctions in which the bids or the reserve price lie above the contemporary price of a brand new iPhone $5(\$ 649)$ sold at the Apple Store (see Wyatt 2013). This leaves a sample of 12,890 auctioned iPhones.

4.3. Estimation Results. Using this data, we estimate, for different carriers and for unlocked phones, the distributions of unobserved heterogeneity, bidder valuations, and reserve prices, where the latter two distributions are net of the unobserved heterogeneity. Therefore, the objects of interest are $F_{U}, F_{W}$, and $F_{X}$. By Theorem 1 , each of these objects is point identified. We note that our method treats bidders in these auctions as though they are short lived, exiting the auction market after one attempt. ${ }^{10}$ We also treat bidders as having private values; as with many auction settings, allowing for interdepencies in valuations would be preferable but would be beyond the state of the methodological literature. However, we believe that the private values assumption is a reasonable approximation to reality here in that all buyers have access to the same information on the website about the product. Also, lemons-like interdependencies - arising from the seller withholding information from the buyer about the quality of the good - are less likely to be a concern in our data than in many other auction settings due to buyer protection plans and sanctions against deceptive sellers, which eBay has incorporated in recent years.

To implement our method, we adopt the log (i.e. multiplicatively separable) specification of the model described at the beginning of Section 2. We account for shipping fees simply by adding them to the observed bids and reserve prices. We then control for observable heterogeneity in the smartphones using the standard homogenization step of Haile, Hong, and Shum (2003) by regressing log reserve prices on observable characteristics, consisting of fully saturated model of indicators for all combinations

\footnotetext{
${ }^{10}$ In practice, this appears to be a reasonable approximation, as $71 \%$ of bidders in our data bid in at most one auction for a given phone specification.
} 
of iPhone model (4 or $4 \mathrm{~S}$ ), memory size $(8,16$, or $32 \mathrm{~GB})$, and color (black or white). ${ }^{11}$ We then compute homogenenized bids and reserve prices by subtracting the regression's predicted value from the log bids and log reserve prices.

With these homogenized bids and reserve prices, we apply the steps described in Section 3 to estimate the model's primitives, estimating $F_{U}, F_{W}$, and $F_{X}$ separately for each carrier code (AT\&T, Verizon, and unlocked) and separately for each time period, with the pre period (prior to the unlocking ban) being September 22, 2012 through January 25, 2013 and the post period (after the unlocking ban took effect) being January 26, 2013 through May 21, 2013.

We begin by estimating the semiparametric model. We specify the marginal densities $f_{U}, f_{W}$, and $f_{X}$, to all be Normal, but we specify $f_{U^{k}}$ to be a fourth-degree Hermite polynomial. This flexibility in the $k^{t h}$ unconditional order statistic density $f_{U^{k}}$ permits the model to accommodate the unknown distribution of the number of bidders. The results of the semiparametric model are displayed in Table 1. Panel A displays the estimated parameters for the pre period and panel B displays the results for the post period.

We find that, in the pre period, the means of buyer valuations and seller reserve prices are higher for unlocked phones than for AT\&T or Verizon phones (although, in the case of buyer valuations for unlocked vs. AT\&T phones, this difference is small in magnitude). This ordering of locked vs. unlocked phones is intuitive, as unlocked phones should obtain a premium given that they can be used on any carrier. The fact that consumers value Verizon phones less than AT\&T phones is likely simply an artifact of Apple phone contracts for Verizon being a relatively new phenomenon during our sample period when compared to such contracts for AT\&T. We find that the standard deviations of seller reserve prices and unobserved heterogeneity are similar across carriers. ${ }^{12}$

\footnotetext{
${ }^{11}$ We perform this homogenization step using using log reserve prices as the left hand side variable, but the log of bids could have be used instead of (or in addition to) the log of reserve prices.

${ }^{12}$ Recall that the mean of the unobserved heterogeneity distribution is mechanically the same for each carrier, but the standard deviation is allowed to differ.
} 
Comparing across the two time periods, we see that several model parameters are quite similar before and after the unlocking ban for all carriers and unlocked phones, including the standard deviation of seller reserve prices and the standard deviation of unobserved heterogeneity. The distributions of buyer valuations and seller reserve prices for AT\&T phones also stayed roughly constant before and after the ban. For Verizon phones, the mean of buyer valuations decreased from -0.287 to $-0.340 \log$ points and the mean of seller reserve prices decreased from -0.072 to $-0.145 \log$ points. For unlocked phones, the mean of seller reserve prices changed only slightly, but the mean of buyer valuations had a much larger shift, dropping from 0.036 to $-0.155 \log$ points after the ban went into affect. The difference of 0.119 is roughly equal to a decrease of 0.5 standard deviations of the estimated distribution of logvaluations, or a decrease in the dollar valuation for the phone of about 11\%. Moreover, the standard deviation increased by roughly $20 \%$.

To examine whether any of the above implications are driven by parametric restrictions, we now turn to the results of the nonparametric model. For this exercise, we specify $f_{U}, f_{W}$, and $f_{U^{k}}$ each as fourth-degree Hermite polynomials. For the density $f_{X}$ we use a third-degree Hermite polynomial. ${ }^{13}$ Figure 1 includes all of the nonparametric estimates for AT\&T, Verizon, and unlocked phones in the pre period, and Figure 2 contains the analogous estimates in the post period.

Figures 1 and 2 suggest, as did the results of the semiparametric model, that the distribution of unobserved heterogeneity is similar for AT\&T, Verizon, and unlocked phones, both before and after the unlocking ban. We also find again that distributions of seller reserve prices have a clear stochastic ordering in both the pre and post periods, with unlocked phones being highest, then AT\&T phones, and then Verizon phones. The ordering for buyer valuations in the pre period is roughly the same, although

\footnotetext{
${ }^{13}$ This latter choice is driven by the fact that $X$, the unobserved heterogeneity, is the dimension over which we integrate in computing the likelihood, and, even with many Gauss-Hermite nodes, there can be few nodes close to zero. With an even-degree polynomial, this can yield an estimated density with little mass exactly at zero and a mode on either side of zero. With a third-degree polynomial approximation, the integration and estimation result in an estimated density that is single-peaked. The qualitative results and the pre and post comparison are similar when all densities are approximated with fourth-degree polynomials. Figures 7 and 8 in the Appendix displays these results.
} 
buyer valuations for AT\&T and unlocked phones are quite similar to one another. In the post period, however, we see that the ordering for buyer valuations changes, with unlocked phones being valued much less than AT\&T phones, although still more than Verizon phones. This shift is suggestive that buyers may have been less willing to pay for technologically circumvented handsets after the unlocking ban took effect.

Figures 3-5 display these same estimates separately by carrier code for the pre vs. post time period. In each of these three figures, the distribution of unobserved heterogeneity is similar pre and post the unlocking ban. In Figure 3 the distributions of buyer valuations for AT\&T phones is slightly lower in the post period, which is likely due in part to the passage of time, depreciating buyers' willingness to pay for a fixed model. The distribution of seller reserve prices for AT\&T phones is similar pre and post. Figure 4 displays a similar decrease in buyer valuations for Verizon phones, and also a decrease in seller valuations, in the post period relative to the pre period. In Figure 5, however, there is a dramatic shift in buyer valuations for unlocked phones. The estimated difference in means of the distributions in the pre and post periods is 0.31, corresponding to about 0.9 standard deviations of the estimated distribution of log-valuations, or a decrease in the dollar valuation for the phone of about $27 \%$. The estimated mean decreases in the dollar valuations for AT\&T and Verizon phones are only $5.1 \%$ and $4.6 \%$, respectively. This is again suggestive evidence that buyers may have incorporated into their valuations a distaste for violations of digital rights laws.

Thus far we have not attempted to identify seller valuations directly, but rather worked only with sellers' reported reserve prices. We now consider the seller valuations that would be implied by the data if the reserve prices are interpreted as having been set optimally by the seller (given the distribution of buyer valuations). As explained in Section 2, if the seller has a valuation that is additively separable in the auction-level heterogeneity, the optimal reserve price (net of auction-level heterogeneity) would satisfy $W=S+\frac{1-F_{U}(W)}{f_{U}(W)}$, and thus the seller's net valuation is given by $S=M R_{U}(W) \equiv W-\frac{1-F_{U}(W)}{f_{U}(W)}$, where the notation $M R_{U}(\cdot)$ denotes the 
buyer's marginal revenue as in Bulow and Roberts (1989). ${ }^{14}$ The distribution of seller valuations is then given by $F_{S}(s)=F_{W}\left(M R_{U}^{-1}(s)\right)$.

We compute the implied seller distributions for AT\&T, Verizon, and unlocked phones in the pre and post periods using the estimated parameters from the semiparametric model from Table $1 .{ }^{15}$ We show these distributions in Figure 6 . We find that implied seller valuations for AT\&T phones, the top panel, increased slightly and implied seller valuations for Verizon phones, the middle panel, decreased slightly from the pre period to the post period. Implied seller valuations for unlocked phones appear nearly identical before and after the unlocking ban. These findings are consistent with our findings above that the primary change in market valuations for these used smartphones occurred on the buyers' side and only for unlocked phones.

\section{Conclusion}

This paper introduces a new approach to identification in auctions with unobserved auction-level heterogeneity and an unknown number of bidders. The methodology relies on deconvolution ideas for handling unobserved heterogeneity - which have been applied extensively to first price auctions but not to ascending auctions due the complicating factor of correlation between order statistics. The approach also relies on order statistics comparisons which have previously been limited to settings that do not allow for unobserved heterogeneity. We bring these ideas together in a unified framework, exploiting information contained in reserve prices - either secret or public - chosen by the seller. We provide point identification as well as partial identification results for these settings and propose a nonparametric sieve maximum likelihood approach or semiparametric approach for estimation and inference.

\footnotetext{
${ }^{14}$ This same property holds in logs for the case where valuations are multiplicatively separable in the unobserved heterogeneity term.

${ }^{15}$ We use the semiparametric rather than nonparametric model to simplify the inversion from observed reserve prices to seller valuations. Specifically, the normality assumption for $F_{U}$ in the semiparametric model ensures that $F_{U}$ is regular in the sense of Myerson (1981), i.e. $\psi(w) \equiv w-\frac{1-F_{U}(w)}{f_{U}(w)}$ will be strictly increasing in $w$ and hence will correspond to marginal revenue. If $\psi(w)$ is not strictly increasing (i.e., the distribution is irregular) then marginal revenue, $M R_{U}(w)$, would instead be computed as the derivative of the convex hull of $\int_{0}^{w} \psi(y) d y$ (the ironed marginal revenue function).
} 
We apply this framework to analyze changes in bidders' willingness to pay and sellers' reserve prices before and after 2013 regulatory changes banning the removal of digital locks on cellphones. We find that, for phones locked to AT\&T or Verizon phones, as well as for unlocked phones, the distributions of unobserved heterogeneity and of seller reserve prices were quite similar before and after the unlocking ban. We also find that the distribution of buyer valuations was similar before and after the ban for AT\&T and Verizon phones. However, for unlocked phones - those which, after the law took effect, were potentially in violation of digital rights legislation - there was a clear drop in buyer valuations, suggestive that digital rights management laws, while difficult to enforce in practice, may have real effects on consumers' willingness to pay.

We believe our methodology has applications to ascending auctions more broadly, whether they be traditional English auctions, such as for timber, cattle, or used cars; or online auctions for e-commerce or display advertising. In many of these settings, unobserved heterogeneity in the auctioned items is a major concern, and the number of bidders is often unknown to the econometrician, as many such auctions do not require all bidders to register in any fashion prior to the auction and hence their would-be bids are unobserved. 


\section{REFERENCES}

Ackerberg, D., X. Chen, and J. Hahn (2012): "A practical asymptotic variance estimator for two-step semiparametric estimators," Review of Economics and Statistics, 94(2), 481-498.

An, Y., Y. Hu, And M. Shum (2010): "Estimating first-price auctions with an unknown number of bidders: A misclassification approach," Journal of Econometrics, 157(2), 328-341.

Aradillas-López, A., A. Gandhi, and D. Quint (2013): "Identification and inference in ascending auctions with correlated private values," Econometrica, 81(2), 489-534.

Armstrong, T. B. (2013): "Bounds in auctions with unobserved heterogeneity," Quantitative Economics, 4(3), 377-415.

Athey, S., And P. A. Haile (2002): "Identification of standard auction models," Econometrica, 70(6), 2107-2140.

(2007): "Nonparametric approaches to auctions," Handbook of Econometrics, 6, 3847-3965.

Athey, S., J. Levin, And E. Seira (2011): "Comparing open and sealed bid auctions: Evidence from timber auctions," The Quarterly Journal of Economics, 126(1), 207-257.

BAKER, C. A. (2007): "Consumer switching costs in the US marketplace for wireless telephone service," Working paper, AARP Public Policy Institute.

BALAT, J. (2015): "Highway procurement and the stimulus package: Identification and estimation of dynamic auctions with unobserved heterogeneity," Working paper, Johns Hopkins University.

(2016): "Identification and estimation of affiliated private values auctions with unobserved heterogeneity," Working paper, Johns Hopkins University.

Bulow, J., AND J. Roberts (1989): "The simple economics of optimal auctions," Journal of Political Economy, pp. 1060-1090.

Canals-Cerdá, J. J., And J. Pearcy (2013): "Arriving in time: Estimation of English auctions with a stochastic number of bidders," Journal of Business \& 
Economic Statistics, 31(2), 125-135.

Chen, X. (2007): "Large sample sieve estimation of semi-nonparametric models," in Handbook of Econometrics, ed. by J. Heckman, and E. Leamer, vol. 6 of Handbook of Econometrics, chap. 76, pp. 5550-5623. Elsevier.

Chen, X., E. T. Tamer, and A. Torgovitsky (2011): "Sensitivity analysis in semiparametric likelihood models," Cowles Foundation Discussion Paper 1836.

Coey, D., B. Larsen, and K. Sweeney (2016): "The bidder exclusion effect," NBER Working Paper 20523.

Coey, D., B. Larsen, K. Sweeney, and C. Waisman (2017): "Ascending auctions with bidder asymmetries," Quantitative Economics, 8(1), 181-200.

Couts, A. (2012): "Unlocking your smartphone will soon be illegal, and other DMCA new rule insanity," Url:http://www.digitaltrends.com/mobile/unlockingyour-smartphone-will-soon-be-illegal-and-other-new-dmca-rule-insanity/. Posted October 26, 2012. Retrieved July 26, 2016.

David, H. A., And H. N. Nagaraja (2003): Order Statistic. New York: Wiley.

DeCarolis, F. (2017): "Comparing public procurement auctions," International Economic Review, forthcoming.

Evdokimov, K., And H. White (2012): "Some extensions of a lemma of Kotlarski," Econometric Theory, 28(4), 925-923.

FAN, Y., AND C. YANG (2016): "Competition, product proliferation and welfare: A study of the US smartphone market," Working paper, University of Michigan.

Farrell, J., And P. Klemperer (2007): "Coordination and lock-in: Competition with switching costs and network effects," Handbook of Industrial Organization, 3, $1967-2072$.

Federal Register (2012): "Exemption to prohibition on circumvention of copyright protection systems for access control technologies," Federal Register, 77(208), Url:http://copyright.gov/fedreg/2012/77fr65260.pdf.

Gallant, A., And D. NyChKa (1987): "Semi-nonparametric maximum likelihood estimation," Econometrica, pp. 363-390. 
Haile, P. A., H. Hong, And M. Shum (2003): "Nonparametric tests for common values at first-price sealed-bid auctions," NBER Working Paper 10105.

Haile, P. A., And E. TAmer (2003): "Inference with an incomplete model of English auctions," Journal of Political Economy, 111(1), 1-51.

Harbaugh, R., and R. Khemka (2010): "Does copyright enforcement encourage piracy?," Journal of Industrial Economics, 58(2), 306-323.

Hickman, B. R., T. P. Hubbard, and H. J. PaArsch (2016): "Identification and estimation of a bidding model for electronic auctions," Quantitative Economics, forthcoming.

Hortacsu, A., and E. R. Nielsen (2010): "Commentary: Do bids equal values on eBay?," Marketing Science, 29(6), 994-997.

Hu, Y., D. McAdams, and M. Shum (2013): "Identification of first-price auctions with non-separable unobserved heterogeneity," Journal of Econometrics, 174(2), $186-193$.

JudD, K. (1998): Numerical Methods in Economics. Cambridge: MIT Press.

Khanna, D. (2013): "The most ridiculous law of 2013 (so far): It is now a crime to unlock your smartphone," Url:https://www.theatlantic.com/business/archive/2013/01/the-most-ridiculouslaw-of-2013-so-far-it-is-now-a-crime-to-unlock-your-smartphone/272552/. Posted January 27, 2013. Retrieved May 30, 2017.

KIm, K. I., AND J. LEE (2014): "Nonparametric estimation and testing of the symmetric IPV framework with unknown number of bidders," Working paper, Michigan State University.

Kotlarski, I. (1967): "On characterizing the Gamma and Normal distribution," Pacific Journal of Mathematics, 20(1), 69-76.

KrasnokUtSkAYA, E. (2011): "Identification and estimation of auction models with unobserved heterogeneity," Review of Economic Studies, 78(1), 293-327.

Krasnokutskaya, E., And K. Seim (2011): "Bid preference programs and participation in highway procurement auctions," American Economic Review, 101(6), 2653-2686. 
LARSEn, B. (2014): "The efficieny of real-world bargaining: Evidence from wholesale used-auto auctions," NBER Working Paper 20431.

Li, T., I. Perrigne, and Q. Vuong (2000): "Conditionally independent private information in OCS wildcat auctions," Journal of Econometrics, 98(1), 129-161.

LI, T., AND Q. VuONG (1998): "Nonparametric estimation of the measurement error model using multiple indicators," Journal of Multivariate Analysis, 65(2), 139-165.

LiU, Q., R. Safavi-Naini, and N. P. Sheppard (2003): "Digital rights management for content distribution," in Proceedings of the Australasian information security workshop conference on ACSW frontiers 2003-Volume 21, pp. 49-58. Australian Computer Society, Inc.

Maicas, J. P., Y. Polo, and F. J. Sese (2009): "Reducing the level of switching costs in mobile communications: The case of mobile number portability," Telecommunications Policy, 33(9), 544-554.

Myerson, R. B. (1981): "Optimal auction design," Mathematics of Operations Research, 6(1), 58-73.

NAKAmurA, A. (2010): "Estimating switching costs involved in changing mobile phone carriers in Japan: Evaluation of lock-in factors related to Japans SIM card locks," Telecommunications Policy, 34(11), 736-746.

PARK, Y., AND Y. KoO (2016): "An empirical analysis of switching cost in the smartphone market in South Korea," Telecommunications Policy, 40(4), 307-318.

Platt, B. (2015): "Inferring ascending auction participation from observed bidders," Working paper, Brigham Young University.

QuiNT, D. (2015): "Identification in symmetric English auctions with additively separable unobserved heterogeneity," Working paper, University of Wisconsin.

RoBerts, J. W. (2013): "Unobserved heterogeneity and reserve prices in auctions," RAND Journal of Economics, 44(4), 712-732.

SAILER, K. (2006): "Searching the eBay marketplace," CESifo Working Paper Series 1848.

Shen, X., And J. Shi (2005): "Sieve likelihood ratio inference on general parameter space," Science in China Series A: Mathematics, 48(1), 67-78. 
Sinkinson, M. (2014): "Pricing and entry incentives with exclusive contracts: Evidence from smartphones," Working paper, University of Pennsylvania.

SonG, U. (2004): "Nonparametric estimation of an eBay auction model with an unknown number of bidders," Working paper, University of British Columbia.

Stallman, R. (1997): "The right to read," Communications of the ACM, 40(2), 85-87.

Tallberg, M., H. Hämmäinen, J. TÖyli, S. Kamppari, and A. Kivi (2007): "Impacts of handset bundling on mobile data usage: The case of Finland," Telecommunications Policy, 31(10), 648-659.

VAn CAmp, J. (2013): "Stick it to the man! Phone unlocking skyrockets 70 percent since becoming illegal," Url:http://www.digitaltrends.com/mobile/phoneunlocking-skyrockets-since-ban/. Posted August 13, 2013. Retrieved September 1, 2016.

Velazco, C. (2013): "Unlocking your phone is now illegal, but what does that mean for you?," Url:https://techcrunch.com/2013/01/26/unlocking-your-phone-isnow-illegal-but-what-does-that-mean-for-you/. Posted January 26, 2013. Retrieved May 30, 2017.

Von Lohmann, F. (2004): "Measuring the Digital Millennium against the Darknet: Implications for the regulation of technological protection measures," Loyola of Los Angeles Entertainment Law Review, 24, 635.

WALKeR, J. (2003): "The digital imprimatur: How big brother and big media can put the Internet genie back in the bottle," Knowledge, Technology, and Policy, 16(3), 24-77.

WyAtT, E. (2013): "FCC backs consumers in unlocking of cellphones," New York Times, p. B1, March 5, 2013.

Zhu, T., H. Liu, and P. K. Chintagunta (2015): "Wireless carriers exclusive handset arrangements: An empirical look at the iPhone," Customer Needs and Solutions, 2(2), 177-190. 
Table 1. Estimated Parameters From Semiparametric Model

\begin{tabular}{|c|c|c|c|}
\hline & AT\&T & Verizon & Unlocked \\
\hline \multicolumn{4}{|l|}{ A. Pre Unlocking Ban } \\
\hline $\begin{array}{l}\text { Mean of buyer valuations }(U) \\
\text { Std of buyer valuations }(U) \\
\text { Mean of seller reserve }(W) \\
\text { Std of seller reserve }(W) \\
\text { Std of unobs heterogeneity }(X)\end{array}$ & $\begin{array}{l}0.032 \\
0.145 \\
0.024 \\
0.240 \\
0.100\end{array}$ & $\begin{array}{c}-0.287 \\
0.220 \\
-0.072 \\
0.221 \\
0.105\end{array}$ & $\begin{array}{l}0.036 \\
0.187 \\
0.183 \\
0.220 \\
0.102\end{array}$ \\
\hline \multicolumn{4}{|l|}{ B. Post Unlocking Ban } \\
\hline $\begin{array}{l}\text { Mean of buyer valuations }(U) \\
\text { Std of buyer valuations }(U) \\
\text { Mean of seller reserve }(W) \\
\text { Std of seller reserve }(W) \\
\text { Std of unobs heterogeneity }(X)\end{array}$ & $\begin{array}{l}0.010 \\
0.166 \\
0.027 \\
0.235 \\
0.104\end{array}$ & $\begin{array}{c}-0.340 \\
0.204 \\
-0.145 \\
0.232 \\
0.106\end{array}$ & $\begin{array}{c}-0.155 \\
0.254 \\
0.167 \\
0.254 \\
0.107\end{array}$ \\
\hline
\end{tabular}

Notes: Table displays estimates from semiparametric model, where densities $f_{U}, f_{W}$, and $f_{X}$ are each Normally distributed and $f_{U^{k}}$ is a fourth-degree Hermite polynomial. Units are log points, after homogenization (i.e. subtracting off observable auction-level heterogeneity). 
Figure 1. Pre Unlocking Ban: Distribution Functions and Densities for AT\&T, Verizon, and Unlocked Phones
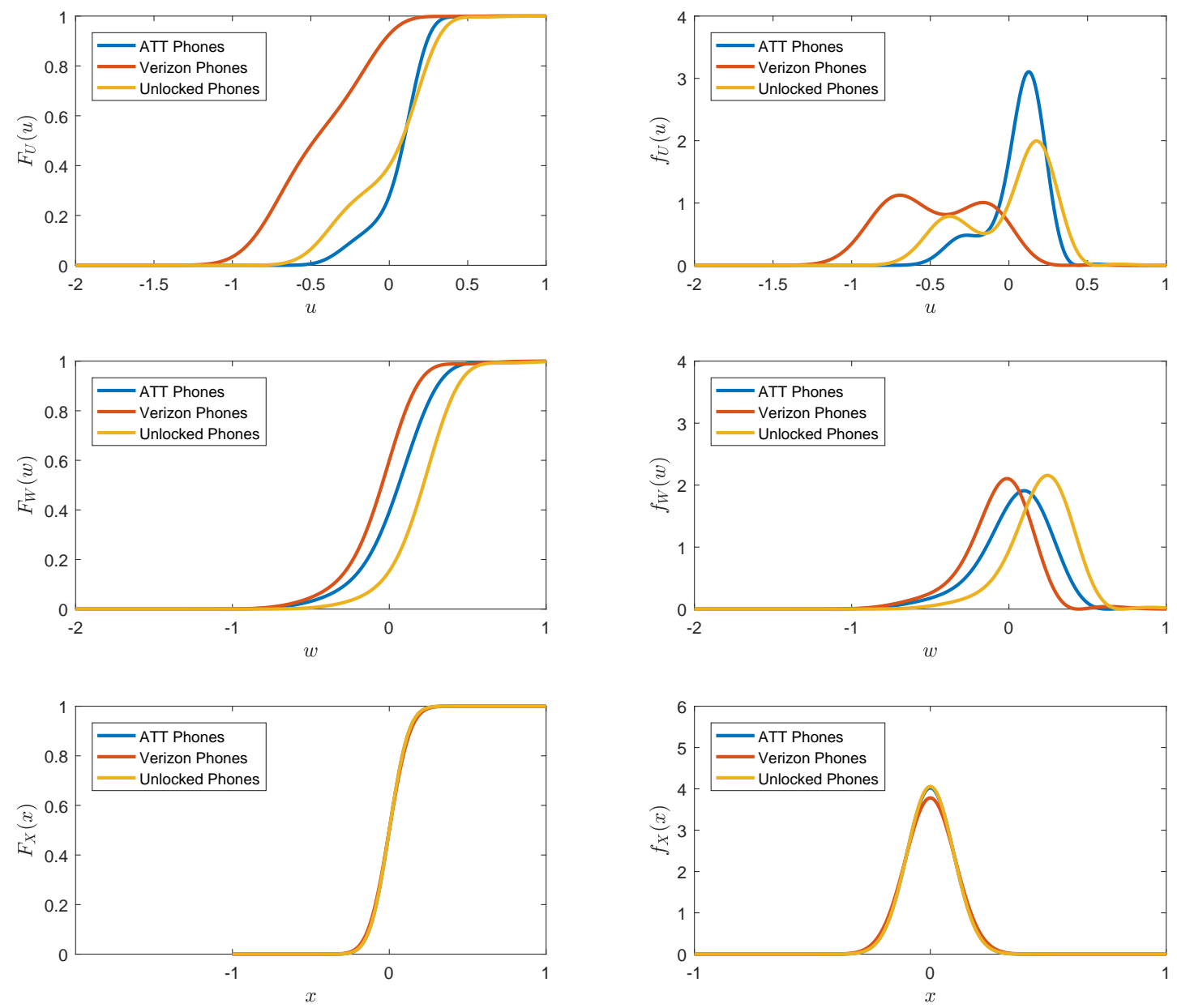

Notes: Each plot shows nonparametric estimates for AT\&T, Verizon, and unlocked phones in the pre period. Panels on the left show distribution functions and on the right, densities, for the noncommon component of buyer valuations $F_{U}$ (top row) and reserve prices $F_{W}$ (middle row), and for the unobserved heterogeneity $F_{X}$ (bottom row). Units on the horizontal axes are log points, after homogenization (i.e. subtracting off observable auction-level heterogeneity). 
Figure 2. Post Unlocking Ban: Distribution Functions and Densities for AT\&T, Verizon, and Unlocked Phones
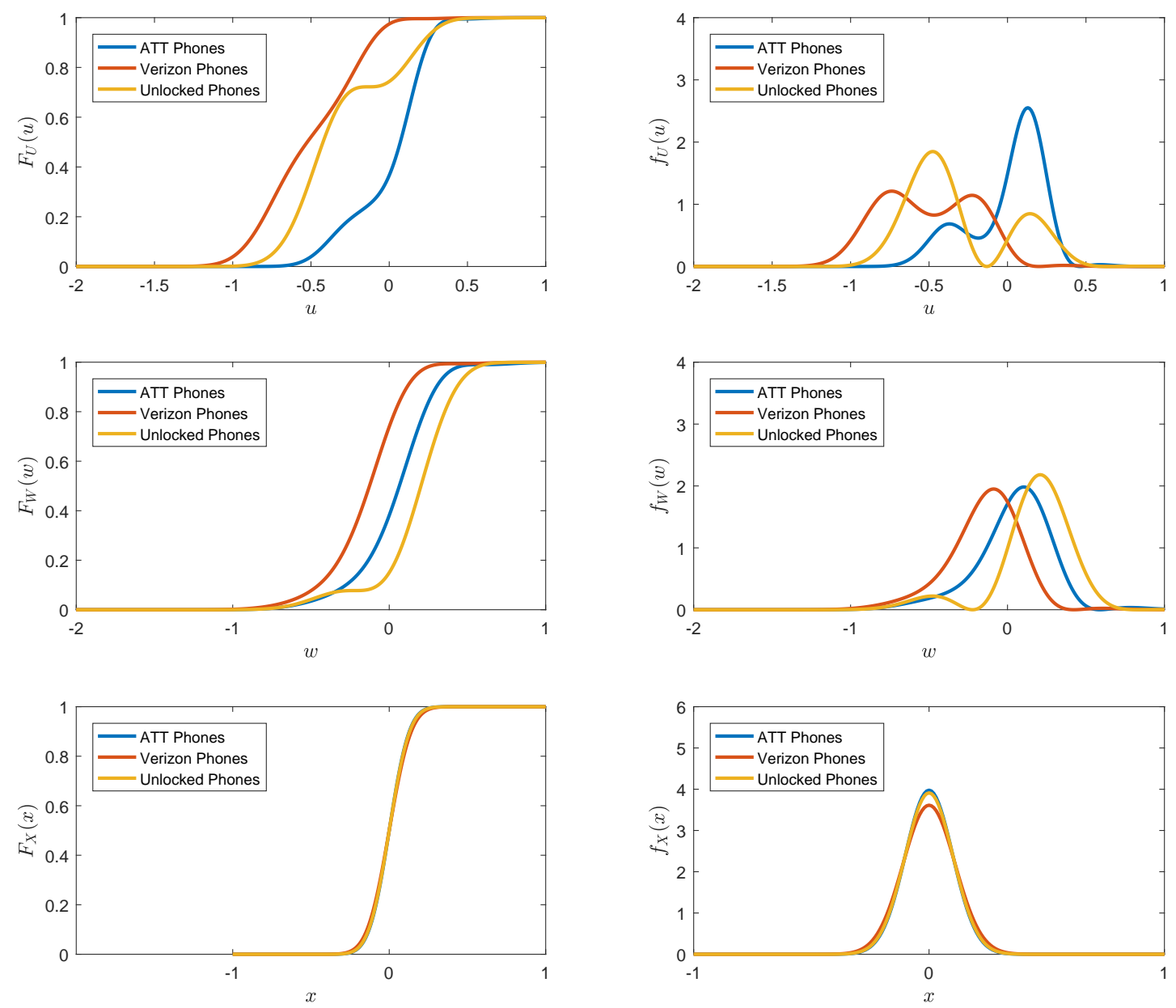

Notes: Each plot shows nonparametric estimates for AT\&T, Verizon, and unlocked phones in the post period. Panels on the left show distribution functions and on the right, densities, for the noncommon component of buyer valuations $F_{U}$ (top row) and reserve prices $F_{W}$ (middle row), and for the unobserved heterogeneity $F_{X}$ (bottom row). Units on the horizontal axes are log points, after homogenization (i.e. subtracting off observable auction-level heterogeneity). 
Figure 3. Estimates for AT\&T-locked iPhones Before and After Unlocking Ban
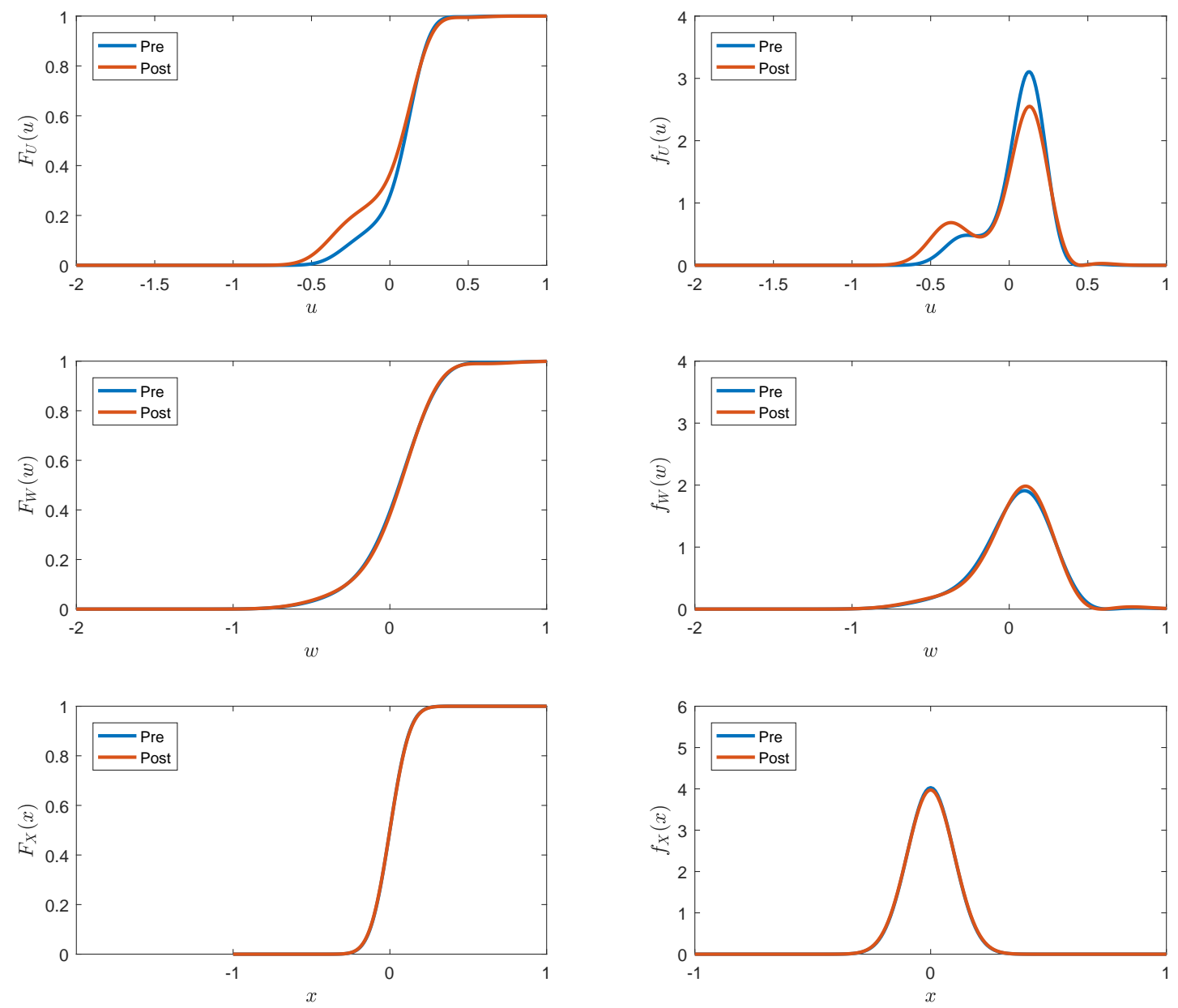

Notes: AT\&T phones only. Each plot shows the nonparametric estimate before and after the regulatory change. Panels on the left show distribution functions and on the right, densities, for the noncommon component of buyer valuations $F_{U}$ (top row) and reserve prices $F_{W}$ (middle row), and for the unobserved heterogeneity $F_{X}$ (bottom row). Units on the horizontal axes are log points, after homogenization (i.e. subtracting off observable auction-level heterogeneity). 
Figure 4. Estimates for Verizon-locked iPhones Before and After Unlocking Ban
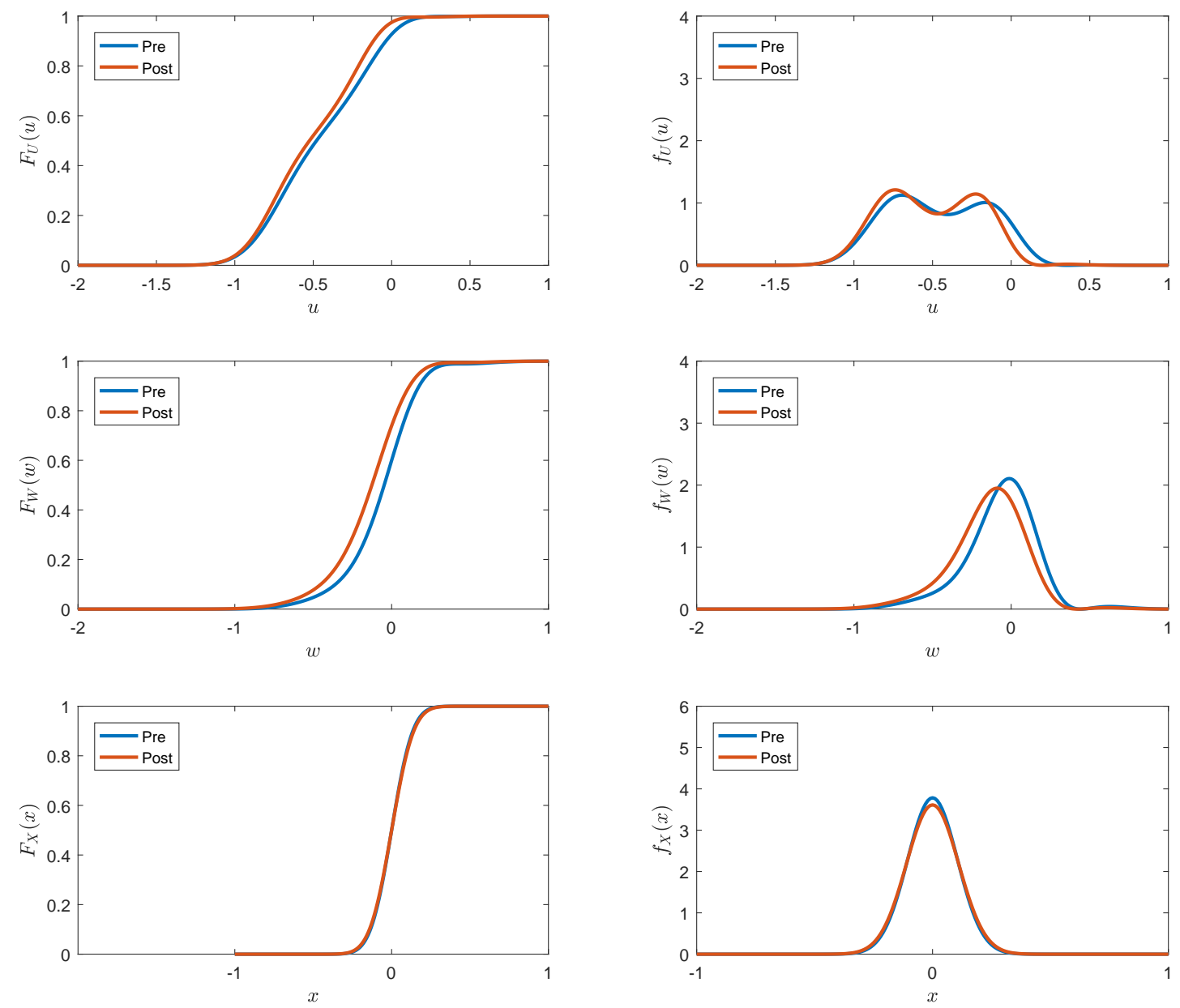

Notes: Verizon phones only. Each plot shows the nonparametric estimate before and after the regulatory change. Panels on the left show distribution functions and on the right, densities, for the noncommon component of buyer valuations $F_{U}$ (top row) and reserve prices $F_{W}$ (middle row), and for the unobserved heterogeneity $F_{X}$ (bottom row). Units on the horizontal axes are log points, after homogenization (i.e. subtracting off observable auction-level heterogeneity). 
Figure 5. Estimates for Unlocked iPhones Before and After Unlocking Ban
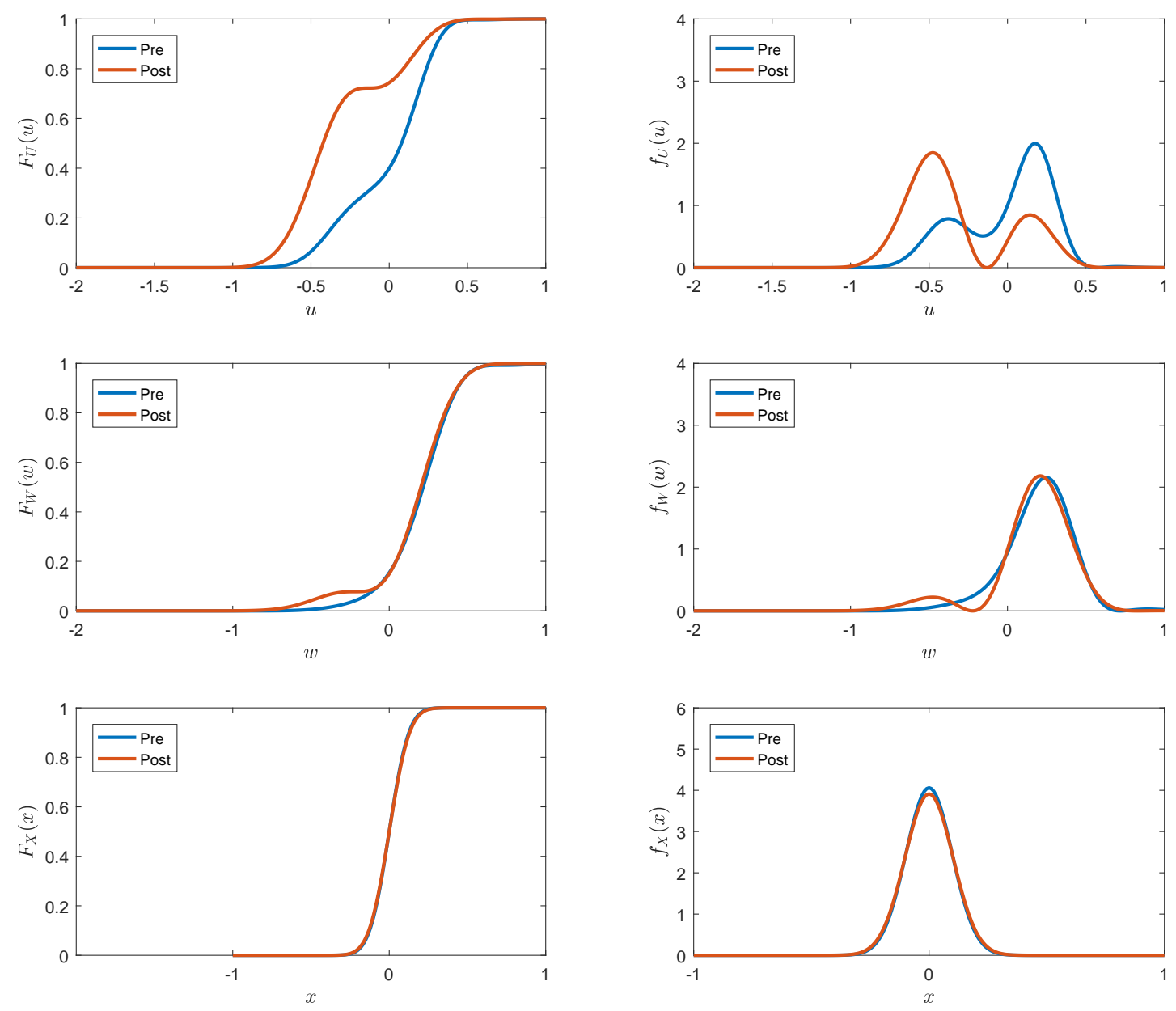

Notes: unlocked phones only. Each plot shows the nonparametric estimate before and after the regulatory change. Panels on the left show distribution functions and on the right, densities, for the noncommon component of buyer valuations $F_{U}$ (top row) and reserve prices $F_{W}$ (middle row), and for the unobserved heterogeneity $F_{X}$ (bottom row). Units on the horizontal axes are log points, after homogenization (i.e. subtracting off observable auction-level heterogeneity). 
Figure 6. Estimates of Implied Seller Valuations Before and After Unlocking Ban, Semiparametric Model
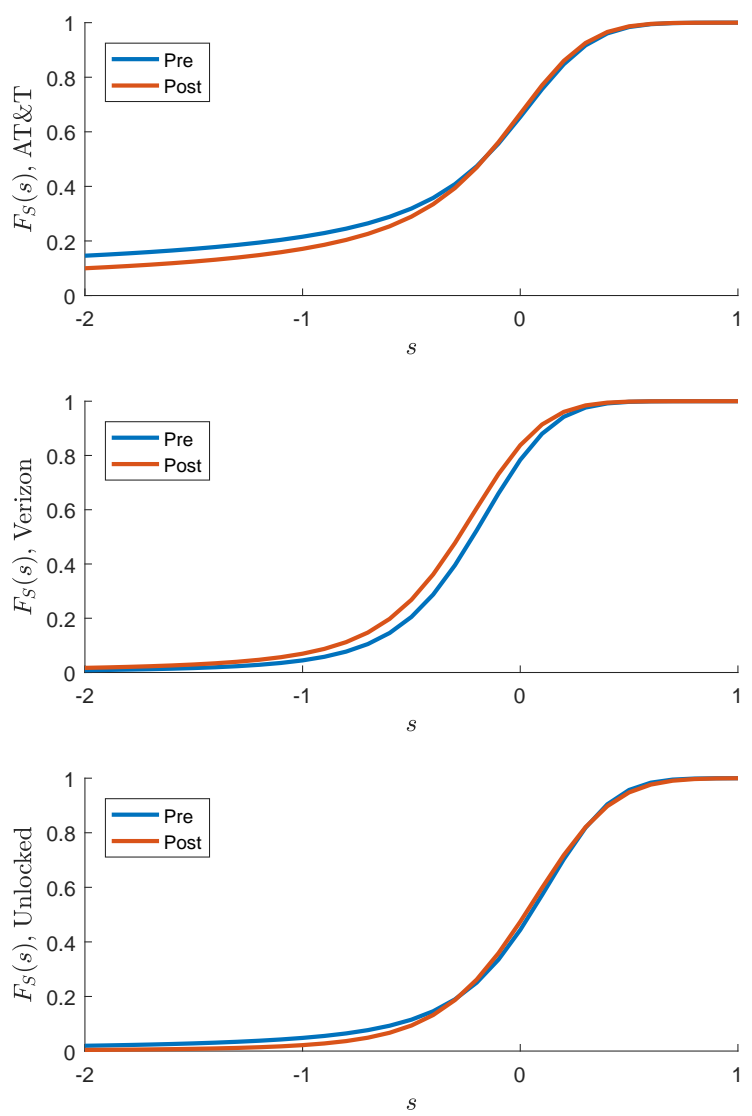

Notes: CDF of seller valuations implied by assuming that seller reserve prices are set optimally. Each plot shows the semiparametric estimate before and after the regulatory change. Upper panel shows AT\&T phones, middle panel shows Verizon phones, and lower panel shows unlocked phones. Units on the horizontal axes are log points, after homogenization (i.e. subtracting off observable auction-level heterogeneity). 
Proof of Theorem 1. First write

$$
\begin{aligned}
B^{j} & =X+U^{j} \\
R & =X+W .
\end{aligned}
$$

By Lemma 1 of Evdokimov and White (2012), $\phi_{X}$ is identified. Moreover, notice that by independence of $X$ and $\left(U^{j}, U^{k}, W\right)$ we get

$$
\phi_{B^{j}, B^{k}, R}\left(t_{j}, t_{k}, t\right)=\phi_{X}\left(t_{j}+t_{k}+t\right) \phi_{U^{j}, U^{k}, W}\left(t_{j}, t_{k}, t\right)
$$

for all $\left(t_{j}, t_{k}, t\right) \in \mathbb{R}^{3}$. Therefore, for all $\left(t_{j}, t_{k}, t\right) \in \mathbb{R}^{3}$ such that $\phi_{X}\left(t_{j}+t_{k}+t\right) \neq 0$,

$$
\phi_{U^{j}, U^{k}, W}\left(t_{j}, t_{k}, t\right)=\frac{\phi_{B^{j}, B^{k}, R}\left(t_{j}, t_{k}, t\right)}{\phi_{X}\left(t_{j}+t_{k}+t\right)} .
$$

It follows that $\phi_{U^{j}, U^{k}, W}\left(t_{j}, t_{k}, t\right)$ is identified for all $\left(t_{j}, t_{k}, t\right) \in \mathbb{R}^{3}$ such that $\phi_{X}\left(t_{j}+\right.$ $\left.t_{k}+t\right) \neq 0$. Since the zeros of $\phi_{X}$ are isolated and since $\phi_{U^{j}, U^{k}, W}\left(t_{j}, t_{k}, t\right)$ is continuous, $\phi_{U^{j}, U^{k}, W}\left(t_{j}, t_{k}, t\right)$ is identified for all $\left(t_{j}, t_{k}, t\right) \in \mathbb{R}^{3}$. Identification of the characteristic function is equivalent to identification of the density $f_{U^{j}, U^{k}, W}$.

Since we know the joint distribution of $U^{j}$ and $U^{k}$, we can use arguments as in Song (2004) to identify the distribution of valuations. Specifically, Song (2004) demonstrated that the density of $U^{j}$ conditional on a realization of $U^{k}$ does not depend on the realization of $N$ :

$$
\begin{aligned}
f_{U^{j} \mid U^{k}}\left(u_{j} \mid u_{k}\right) & =\frac{f_{U^{j}, U^{k}}\left(u_{j}, u_{k}\right)}{f_{U^{k}}\left(u_{k}\right)} \\
& =\frac{(k-1) !\left(F_{U}\left(u_{j}\right)-F_{U}\left(u_{k}\right)\right)^{k-j-1}\left(1-F_{U}\left(u_{j}\right)\right)^{j-1} f_{U}\left(u_{j}\right)}{(k-j-1) !(j-1) !\left(1-F_{U}\left(u_{k}\right)\right)^{k-1}} .
\end{aligned}
$$

Letting $u_{k}$ converge to the lower bound of the support of $U$ identifies

$$
\frac{(k-1) ! F_{U}\left(u_{j}\right)^{k-j-1}\left(1-F_{U}\left(u_{j}\right)\right)^{j-1} f_{U}\left(u_{j}\right)}{(k-j-1) !(j-1) !},
$$


which is the density of the $(j-k)$ 'th order statistic from a sample of size $(j-1)$ with distribution function $F_{U}$. Hence, from Theorem 1 in Athey and Haile (2002), $F_{U}$ is identified.

Finally, note that if $N \in\{\underline{n}, \ldots, \bar{n}\}$ with $\underline{n} \geq k$ and $\bar{n}<\infty$, then

$$
F_{U^{k}}(u)=\sum_{n=\underline{n}}^{\bar{n}} \operatorname{Pr}(N=n)\left[\sum_{i=n-k+1}^{n}\left(\begin{array}{l}
n \\
i
\end{array}\right) F_{U}(u)^{i}\left(1-F_{U}(u)\right)^{n-i}\right]
$$

and therefore for all $z \in[0,1]$

$$
F_{U^{k}}\left(F^{-1}(z)\right)=\sum_{n=\underline{n}}^{\bar{n}} \operatorname{Pr}(N=n)\left[\sum_{i=n-k+1}^{n}\left(\begin{array}{l}
n \\
i
\end{array}\right) z^{i}(1-z)^{n-i}\right]
$$

The terms $\left[\sum_{i=n-k+1}^{n}\left(\begin{array}{c}n \\ i\end{array}\right) z^{i}(1-z)^{n-i}\right]$ are polynomials of order $n$. Hence, the term in $F_{U^{k}}\left(F^{-1}(z)\right)$ belonging to the highest order polynomial, $z^{\bar{n}}$, is

$$
z^{\bar{n}} \operatorname{Pr}(N=\bar{n}) \sum_{i=\bar{n}-k+1}^{\bar{n}}\left(\begin{array}{c}
\bar{n} \\
i
\end{array}\right) F_{U}(u)^{\bar{n}}(-1)^{\bar{n}-i}
$$

Therefore, if $\sum_{i=\bar{n}-k+1}^{\bar{n}}\left(\begin{array}{c}\bar{n} \\ i\end{array}\right)(-1)^{\bar{n}-i} \neq 0$, then $\operatorname{Pr}(N=\bar{n})$ is identified from the $\bar{n}$ 'th derivative of $F_{U^{k}}\left(F^{-1}(z)\right)$ with respect to $z$. Indeed,

$$
\begin{aligned}
\sum_{i=\bar{n}-k+1}^{\bar{n}}\left(\begin{array}{c}
\bar{n} \\
i
\end{array}\right)(-1)^{\bar{n}-i} & =\sum_{j=1}^{k-1}\left(\begin{array}{c}
\bar{n} \\
\bar{n}-j
\end{array}\right)(-1)^{j} \\
& =\sum_{j=1}^{k-1}\left(\begin{array}{c}
\bar{n} \\
j
\end{array}\right)(-1)^{j} \\
& =(-1)^{k-1}\left(\begin{array}{c}
\bar{n}-1 \\
k-1
\end{array}\right) \\
& \neq 0
\end{aligned}
$$

Given identification of $\operatorname{Pr}(N=\bar{n})$, we know

$$
F_{U^{k}}\left(F^{-1}(z)\right)-\operatorname{Pr}(N=\bar{n})\left[\sum_{i=\bar{n}-k+1}^{\bar{n}}\left(\begin{array}{c}
\bar{n} \\
i
\end{array}\right) z^{i}(1-z)^{\bar{n}-i}\right],
$$


which equals

$$
\sum_{n=\underline{n}}^{\bar{n}-1} \operatorname{Pr}(N=n)\left[\sum_{i=n-k+1}^{n}\left(\begin{array}{l}
n \\
i
\end{array}\right) z^{i}(1-z)^{n-i}\right] .
$$

Analogous arguments as above now show identification of $\operatorname{Pr}(N=\bar{n}-1)$. Repeating these steps yield identification of $P(N=n)$ for all $n \in\{\underline{n}, \ldots, \bar{n}\}$.

Proof of Theorem 2. Since $\bar{B} \geq \bar{R}, \bar{B}$ is identified from the largest possible bid. Notice that if $B^{j}=\bar{B}$, then $U^{j}=\bar{U}$ and $X=\bar{X}$. We can now observe for all $r \in[\underline{R}, \bar{R}]=[\underline{X}+\underline{W}, \bar{X}+\bar{W}]$

$$
\begin{aligned}
P\left(R \leq r \mid B^{j}=\bar{B}\right) & =P\left(R \leq r \mid X=\bar{X}, U^{j}=\bar{U}\right) \\
& =P(W \leq r-\bar{X}) .
\end{aligned}
$$

It follows that the distribution of $\tilde{W}=W+\bar{X}$ is identified. But since $E[\tilde{W}]=$ $E[W]+\bar{X}=E[R]+\bar{X}$ and since $E[R]$ is identified, it follows that $\bar{X}$ and hence the distribution of $W$ is identified. By independence of $X$ and $W$,

$$
\phi_{R}(t)=\phi_{X}(t) \phi_{W}(t)
$$

and for all $t$ with $\phi_{W}(t) \neq 0$,

$$
\phi_{X}(t)=\frac{\phi_{R}(t)}{\phi_{W}(t)}
$$

Since $\phi_{R}(t)$ and $\phi_{W}(t)$ are identified and since $\phi_{W}(t)$ has only isolated zeros, $\phi_{X}(t)$ is identified. Thus, $F_{X}$ is identified.

Moreover,

$$
\begin{aligned}
P\left(B^{j} \geq b_{j}, B^{k} \geq b_{k} \mid R=\underline{R}\right) & =P\left(B^{j} \geq b_{j}, B^{k} \geq b_{k} \mid X+W=\underline{X}+\underline{W}\right) \\
& =P\left(B^{j} \geq b_{j}, B^{k} \geq b_{k} \mid X=\underline{X}, W=\underline{W}\right) \\
& =P\left(U^{j} \geq b_{j}-X, U^{k} \geq b_{k}-X \mid X=\underline{X}, W=\underline{W}\right) \\
& =P\left(U^{j} \geq b_{j}-\underline{X}, U^{k} \geq b_{k}-\underline{X}\right)
\end{aligned}
$$


Given $X=\underline{X}$, the support of the observed $B^{j}$ is $[\underline{X}+\underline{W}, \underline{X}+\bar{U}]$. Therefore, if $\underline{B} \geq \underline{R}$ (and thus $\underline{U} \geq \underline{W}$ ), we can identify

$$
P\left(U^{j} \geq u_{j}, U^{k} \geq u_{k}\right)=P\left(B^{j} \geq u_{j}+\underline{X}, B^{k} \geq u_{k}+\underline{X} \mid R=\underline{R}\right) .
$$

for all $u_{j}, u_{k} \in[\underline{U}, \bar{U}]$. In this case, just as in the proof of Theorem 1 , identification of $F_{U^{j}, U^{k}}$ yields identification of $F_{U}$ and $P(N=n)$.

If instead $\underline{B}<\underline{R}$, we can only identify $P\left(U^{j} \geq u_{j}, U^{k} \geq u_{k}\right)$ for all $u_{j} \geq u_{k} \geq \underline{W}>$ $\underline{U}$. Analogous arguments identify $P\left(U^{j} \geq u_{j}\right)$ and $P\left(U^{k} \geq u_{k}\right)$ for all $u_{j}, u_{k} \geq \underline{W}>$ $\underline{U}$. Now since

$$
F_{U^{j} \mid U^{k}}\left(u_{j} \mid u_{k}\right)=1-\int_{u_{j}}^{\bar{U}} \frac{f_{U^{j}, U^{k}}\left(z, u_{k}\right)}{f_{U^{k}}\left(u_{k}\right)} d z,
$$

$F_{U^{j} \mid U^{k}}\left(u_{j} \mid u_{k}\right)$ for all $u_{j} \geq u_{k} \geq \underline{W}$ is identified as well. Therefore, by the arguments from Song (2004), knowing $F_{U^{j} \mid U^{k}}\left(u_{j} \mid u_{k}\right)$ implies knowledge of

$$
\left(F_{U}\left(u_{j}\right)-F_{U}\left(u_{k}\right)\right) /\left(1-F_{U}\left(u_{k}\right)\right)
$$

for all $u_{j} \geq u_{k} \geq \underline{W}$. In other words, we can identify $P\left(U \leq u_{j} \mid U \geq u_{k}\right)$ for all $u_{j}, u_{k} \geq \underline{W}$.

\section{Appendix B. Likelihood Derivation}

Here we derive the expression for $p_{1}, p_{2}$, and $p_{3}$ given in Section 3.2. For the first part write

$$
\begin{aligned}
P\left(R \leq r, D_{1}=1\right) & =P\left(R \leq r, B^{k} \leq B^{j}<R\right) \\
& =P\left(R \leq r, B^{j}<R\right) \\
& =\int_{-\infty}^{\infty} P\left(W \leq r-x, U^{j} \leq W\right) f_{X}(x) d x \\
& =\int_{-\infty}^{\infty} \int_{-\infty}^{\infty} P\left(W \leq r-x, u \leq W \mid U^{j}=u_{j}\right) f_{U^{j}}\left(u_{j}\right) f_{X}(x) d u_{j} d x \\
& =\int_{-\infty}^{\infty} \int_{-\infty}^{r-x} P\left(u_{j} \leq W \leq r-x\right) f_{U^{j}}\left(u_{j}\right) f_{X}(x) d u_{j} d x \\
& =\int_{-\infty}^{\infty} \int_{-\infty}^{r-x}\left(P(W \leq r-x)-P\left(W \leq u_{j}\right)\right) f_{U^{j}}\left(u_{j}\right) f_{X}(x) d u d x
\end{aligned}
$$


Taking the derivative with respect to $r$ (using Leibniz's rule) yields

$$
\begin{aligned}
p_{1}(r) & =\int_{-\infty}^{\infty} \int_{-\infty}^{r-x} f_{W}(r-x) f_{U^{j}}\left(u_{j}\right) f_{X}(x) d u_{j} d x \\
& =\int_{-\infty}^{\infty} F_{U^{j}}(r-x) f_{W}(r-x) f_{X}(x) d x
\end{aligned}
$$

If the number of bidders is unknown, we need expressions in terms of the distributions of $U^{j} \mid U^{k}$ and $U^{k}$ and we use

$$
F_{U^{j}}\left(u_{j}\right)=\int_{-\infty}^{-\infty} F_{U^{j} \mid U^{k}}\left(u_{j} \mid u_{k}\right) f_{U^{k}}\left(u_{k}\right) d u_{k} .
$$

Specifically, when $B^{j}$ is the second highest and $B^{k}$ is the third highest bid, then

$$
f_{U^{j} \mid U^{k}}\left(u_{j}, \mid u_{k}\right)=\frac{2\left(1-F_{U}\left(u_{j}\right)\right) f_{U}\left(u_{j}\right)}{\left(1-F_{U}\left(u_{k}\right)\right)^{2}} .
$$

Thus,

$$
\begin{aligned}
F_{U^{j} \mid U^{k}}\left(u_{j}, \mid u_{k}\right) & =\int_{u_{k}}^{u_{j}} \frac{2\left(1-F_{U}(z)\right) f_{U}(z)}{\left(1-F_{U}\left(u_{k}\right)\right)^{2}} d z \\
& =\frac{\left(2 F_{U}\left(u_{j}\right)-F_{U}\left(u_{j}\right)^{2}\right)-\left(2 F_{U}\left(u_{k}\right)-F_{U}\left(u_{k}\right)^{2}\right)}{\left(1-F_{U}\left(u_{k}\right)\right)^{2}} \mathbf{1}\left(u_{j} \geq u_{k}\right)
\end{aligned}
$$

Similarly,

$$
\begin{aligned}
P\left(B^{j}\right. & \left.\leq b_{j}, R \leq r, D_{2}=1\right) \\
& =P\left(B^{j} \leq b_{j}, R \leq r, B^{j} \geq R>B^{k}\right) \\
& =\int_{-\infty}^{\infty} P\left(U^{j} \leq b_{j}-x, W \leq r-x, U^{j} \geq W>U^{k}\right) f_{X}(x) d x \\
& =\int_{-\infty}^{\infty} \int_{-\infty}^{\infty} P\left(U^{j} \leq b_{j}-x, w \leq r-x, U^{j} \geq w>U^{k} \mid W=w\right) f_{W}(w) f_{X}(x) d u d x \\
& =\int_{-\infty}^{\infty} \int_{-\infty}^{\min \left\{b_{j}-x, r-x\right\}} P\left(w \leq U^{j} \leq b_{j}-x, U^{k}<w\right) f_{W}(w) f_{X}(x) d w d x \\
& =\int_{-\infty}^{\infty} \int_{-\infty}^{\min \left\{b_{j}-x, r-x\right\}}\left(P\left(U^{j} \leq b_{j}-x, U^{k}<w\right)-P\left(U^{j} \leq w, U^{k}<w\right)\right) f_{W}(w) f_{X}(x) d w d x
\end{aligned}
$$


If $b_{j}<r$, then $P\left(B^{j} \leq b_{j}, R \leq r, D_{2}=1\right)$ does not depend on $r$ and $p_{2}\left(r, b_{j}\right)=0$. If $b_{j} \geq r$, then

$$
\begin{aligned}
p_{2}(b, r) & =\frac{\partial}{\partial r \partial b_{j}} P\left(B^{j} \leq b_{j}, R \leq r, D_{2}=1\right) \\
& =\int_{-\infty}^{\infty} \frac{\partial}{\partial b_{j}} P\left(U^{j} \leq b_{j}-x, U^{k}<r-x\right) f_{W}(r-x) f_{X}(x) d x \\
& =\int_{-\infty}^{\infty} F_{U^{k} \mid U^{j}}\left(r-x \mid b_{j}-x\right) f_{U^{j}}\left(b_{j}-x\right) f_{W}(r-x) f_{X}(x) d x
\end{aligned}
$$

Alternatively in terms of the distributions of $U^{j} \mid U^{k}$ and $U^{k}$ write

$$
\begin{aligned}
\frac{\partial}{\partial b_{j}} P\left(U^{j} \leq b_{j}-x, U^{k}<r-x\right) & =\frac{\partial}{\partial b_{j}} \int_{-\infty}^{b_{j}-x} \int_{-\infty}^{r-x} f_{U^{j}, U^{k}}\left(u_{j}, u_{k}\right) d u_{j} d u_{k} \\
& =\int_{-\infty}^{r-x} f_{U^{j}, U^{k}}\left(b_{j}-x, u_{k}\right) d u_{k} \\
& =\int_{-\infty}^{r-x} f_{U^{j} \mid U^{k}}\left(b_{j}-x \mid u_{k}\right) f_{U^{k}}\left(u_{k}\right) d u_{k} .
\end{aligned}
$$

Then

$$
p_{2}\left(r, b_{j}\right)=\int_{-\infty}^{\infty} \int_{-\infty}^{r-x} f_{U^{j} \mid U^{k}}\left(b_{j}-x \mid u_{k}\right) f_{U^{k}}\left(u_{k}\right) d u_{k} f_{W}(r-x) f_{X}(x) d x .
$$

Finally, using the same arguments as before

$$
\begin{aligned}
p_{3}\left(r, b_{j}, b_{2}\right) & =\int_{-\infty}^{\infty} f_{U^{j}, U^{k}}\left(b_{j}-x, b_{k}-x\right) f_{W}(r-x) f_{X}(x) d x \\
& =\int_{-\infty}^{\infty} f_{U^{j} \mid U^{k}}\left(b_{j}-x \mid b_{k}-x\right) f_{U^{k}}\left(b_{k}-x\right) f_{W}(r-x) f_{X}(x) d x
\end{aligned}
$$


Figure 7. Pre Unlocking Ban: Distribution Functions and Densities for AT\&T, Verizon, and Unlocked Phones, with Fourth Degree Hermite Polynomial for Distribution of $X$
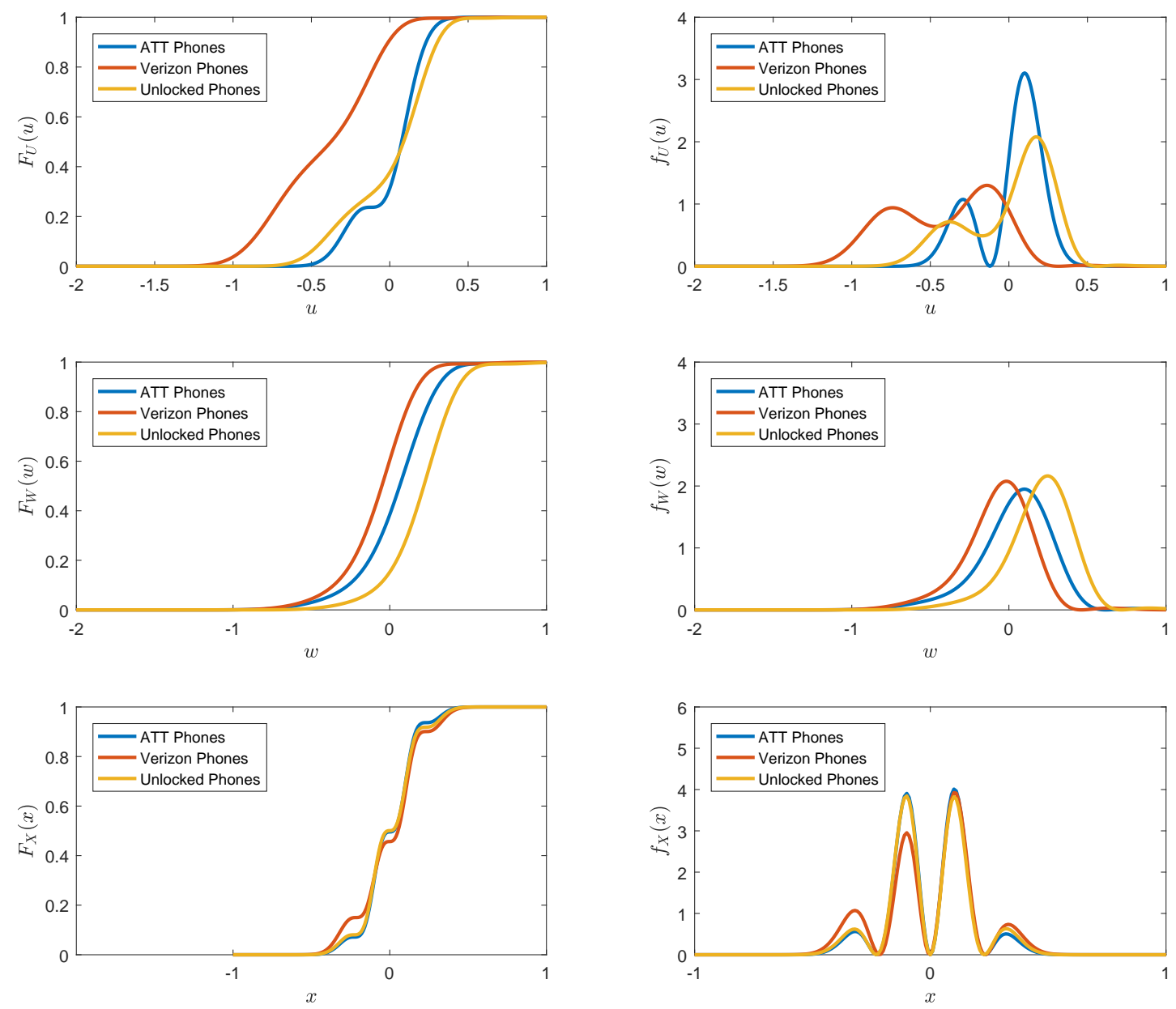

Notes: Each plot shows nonparametric estimates for AT\&T, Verizon, and unlocked phones in the pre period. Panels on the left show distribution functions and on the right, densities, for the noncommon component of buyer valuations $F_{U}$ (top row) and reserve prices $F_{W}$ (middle row), and for the unobserved heterogeneity $F_{X}$ (bottom row). Units on the horizontal axes are log points, after homogenization (i.e. subtracting off observable auction-level heterogeneity). Estimates in this figure use a fourth degree polynomial for $X$ rather than a third-degree polynomial as in Figure 1. 
Figure 8. Post Unlocking Ban: Distribution Functions and Densities for AT\&T, Verizon, and Unlocked Phones, with Fourth Degree Hermite Polynomial for Distribution of $X$
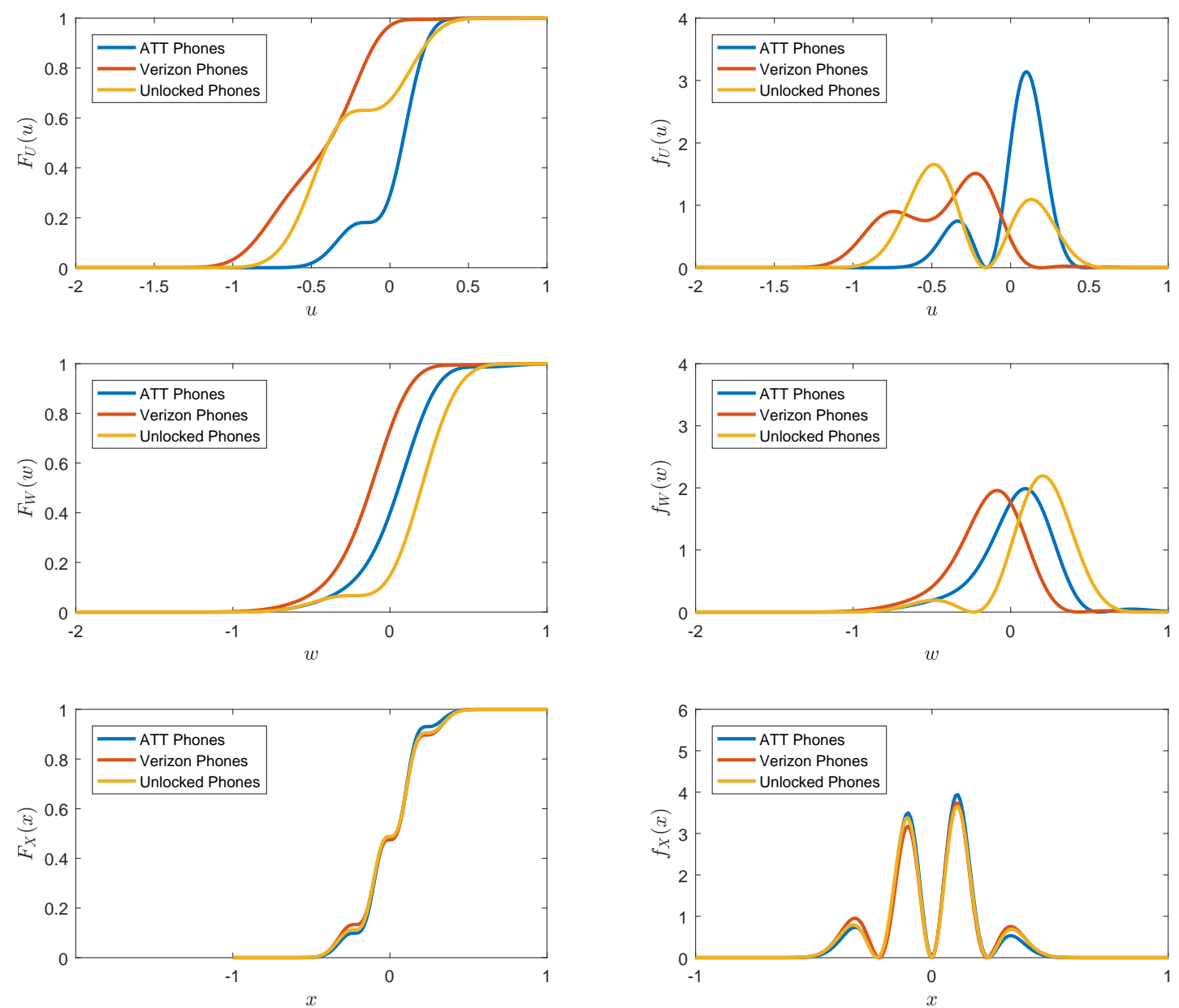

Notes: Each plot shows nonparametric estimates for AT\&T, Verizon, and unlocked phones in the post period. Panels on the left show distribution functions and on the right, densities, for the noncommon component of buyer valuations $F_{U}$ (top row) and reserve prices $F_{W}$ (middle row), and for the unobserved heterogeneity $F_{X}$ (bottom row). Units on the horizontal axes are log points, after homogenization (i.e. subtracting off observable auction-level heterogeneity). Estimates in this figure use a fourth degree polynomial for $X$ rather than a third-degree polynomial as in Figure 2 . 